School of Finance

University of St.Gallen

\title{
A JACKKNIFE-TYPe Estimator FOR PORTFOlio REVISION
}

ROLAND FÜSS

FELIX Miebs

FABIAN TRÜBENBACH

WORKING PAPERS ON FINANCE No. 172

SWISS INSTITUTE OF BANKING AND FINANCE (S/BF - HSG)

MAY 2011 


\title{
A Jackknife-Type Estimator for Portfolio Revision*
}

\author{
Roland Füss ${ }^{\dagger} \quad$ Felix Miebs ${ }^{\ddagger} \quad$ Fabian Trübenbach ${ }^{\S}$
}

March 5, 2013

\begin{abstract}
This paper proposes a novel approach to portfolio revision. The current literature on portfolio optimization uses a somewhat naïve approach, where portfolio weights are always completely revised after a predefined fixed period. However, one shortcoming of this procedure is that it ignores parameter uncertainty in the estimated portfolio weights, as well as the biasedness of the in-sample portfolio mean and variance as estimates of the expected portfolio return and out-of-sample variance. To rectify this problem, we propose a Jackknife procedure to determine the optimal revision intensity, i.e. the percent of wealth that should be shifted to the new, in-sample optimal portfolio. We find that our approach leads to highly stable portfolio allocations over time, and can significantly reduce the turnover of several well established portfolio strategies. Moreover, the observed turnover reductions lead to statistically and economically significant performance gains in the presence of transaction costs.
\end{abstract}

\section{JEL Classification: $G 11$}

Keywords: Portfolio optimization; optimal portfolio revision; out-of-sample performance evaluation; Jackknife estimator; transaction costs

*Acknowledgement: We are grateful to Sebastian Heiden, Alexander Kempf, Kagba Kousse, and the participants of the European Financial Management Symposium 2012 - Asset Management and the 2012 Annual Meeting of the German Finance Association. The opinions expressed in this article are those of the authors. They do not reflect in any way those of the institutions to which they are affiliated. We alone are responsible for any errors.

$\dagger$ Corresponding author: University of St.Gallen, Institute of Banking and Finance (s/bf), Rosenbergstrasse 52, 9000 St. Gallen, Switzerland; Phone: +41 (0)71 224 7010; Fax: +41 (0)71 2247088 ; Email: roland.fuess@unisg.edu.

${ }_{\ddagger}^{\ddagger}$ EBS Business School, Department of Finance, Accounting and Real Estate, Gustav-Stresemann-Ring 3, 65189 Wiesbaden, Germany; Email: felix.miebs@ebs.edu.

$\S$ Macquarie Capital (Europe) Limited, Untermainanlage 1, 60329 Frankfurt, Germany; Email: fabian.truebenbach@macquarie.com. 


\section{Introduction}

Despite the theoretical appeal of Markowitz's (1952) mean-variance paradigm, it has three prominent criticisms. First, the mean-variance algorithm yields highly unbalanced allocations (see, e.g., Green and Hollifield $(1992)$ as well as Black and Litterman (1992)). Second, the impact of even small changes in the input parameters can cause dramatic shifts in the derived allocations (see, e.g., Best and Grauer (1991), Chopra (1993), and Chopra and Ziemba (1993)). Third, its repeated use over time leads to comparatively unstable allocations, resulting in higher turnovers (see Chopra et al. (1993)). The literature contains several potential remedies for the first two issues; relatively little attention, however, has focused on the immense turnover of mean-variance portfolios and the associated question of portfolio revisions. This is the more remarkable as a reduction in turnover involves a riskless increase in the portfolio return in presents of transaction costs. In this paper, we propose a portfolio revision policy that allows investors to control returns after transaction costs, i.e. to earn a risk-free rate without bearing additional (estimation) risk.

By tackling the question of optimal portfolio revision, we explicitly explore when and how much investors should consider shifting from their current portfolios to new insample optimal portfolios. We assess this question based on the established rolling sample approach to portfolio optimization, in which investors typically revise their entire portfolios after a predefined but arbitrarily chosen time period. The investor then holds the new optimal portfolio, which is optimized over the corresponding new in-sample period. This revision policy appears intuitive, because the new portfolio dominates the old one in terms of in-sample mean-variance efficiency. The fixed portfolio revision frequency is also in line with the myopic nature of the Markowitz framework.

However, a significant shortcoming of this approach is that it is based on in-sample mean-variance efficiency. As Kan and Zhou (2007), Siegel and Woodgate (2007), and Basak et al. (2009) have shown, in-sample portfolio mean and variance are not consistent estimators of expected out-of-sample return and variance because of the potential for 
estimation errors. Specifically, these authors note that in-sample portfolio returns that are optimized in the presence of estimation errors tend to overestimate expected out-ofsample returns. In-sample variance, on the other hand, tends to underestimate expected out-of-sample variance $\mathbb{1}^{1}$

As a result of this in-sample overoptimism, investors may be reluctant to revise their portfolios without a concurrent increase in expected out-of-sample performance. To overcome this issue, we base our revision policy on the expected out-of-sample performance of a portfolio, which we consider as the natural investor objective for portfolio revisions.

We use Basak et al.'s (2009) Jackknife procedure to obtain consistent estimates of the expected out-of-sample portfolio performance of the investor's current portfolio versus the new, in-sample optimal portfolio. We then determine what percentage to allocate to the new portfolio. We provide an analytic expression for the optimal revision intensity, and evaluate our revision policy by applying it to various minimum-variance portfolio strategies across five datasets. We could also apply our portfolio revision framework to mean-variance portfolios, but our focus is solely on minimum-variance portfolios because broad empirical evidence has shown that they perform better out-of-sample (see Jobson and Korkie (1981) as well as Jagannathan and Ma (2003), among others).

We assess the performance of our revision policy on simulated as well as on five empirical datasets. For both, simulation and empirical analysis, we achieve comparable portfolio performance in terms of the Sharpe ratio and the certainty equivalent return, suggesting our revision policy can lead to substantially lower turnovers than policies with fixed revision frequencies. Empirically, we observe that our revision policy reduces monthly turnovers by up to $77.6 \%$, with average reductions across all datasets ranging from $15.2 \%$ for the Ledoit and Wolf (2004a) strategy to $66.5 \%$ for the sample based minimum-variance portfolio.

\footnotetext{
${ }^{1}$ Kan and Zhou (2007) as well as Siegel and Woodgate (2007) demonstrate that this in-sample overoptimism is inversely related to the number of observations available to estimate the input parameters for the optimization. It also increases with the number of assets.
} 
If we include trading costs in our analysis, we observe that, because of the sizable turnover reduction, our portfolio revision policy yields economically and statistically significant performance gains. Assuming proportional round-trip transaction costs of $1 \%$, we find that, compared to monthly or annual portfolio revision, investors with a risk aversion of five would theoretically be willing to pay an annual management fee of between 101 and 168 basis points (bps). Our results are especially strong for datasets with larger asset universes, where the higher ratio of number of assets to number of time periods $(N / T)$ increases the estimation error of the covariance matrix estimates. This creates more unbalanced allocations, along with higher turnover.

There are two main strands of literature on portfolio revisions of mean-variance efficient portfolios: dynamic revision strategies (e.g., Chen et al. (1971), Kamin (1975), and Leland (1999) ), and retrospectively determined optimal revision frequencies or ranges (e.g., Arnott and Lovell (1993), Plaxco and Arnott (2002), and Tokat and Wicas (2007)). Dynamic revision strategies are generally complex, computationally intensive, and thus somewhat limited to small investment universes (see Donhue and Yip (2003)). Notably, the Markowitz and van Dijk (2003) as well as Kritzman et al. (2009) approximations represent scalable alternatives to dynamic revision strategies. However, they remain a heuristic approximation to the underlying dynamic program. The heuristic nature of these approximations is also reflected in the retrospectively determined optimal revision frequencies or ranges. The revision frequencies do not follow any objective optimality criteria, and may thus be regarded as heuristic.

In addition to the two major approaches to portfolio revisions, the literature contains two minor approaches: the imposition of turnover constraints (Schreiner (1980)), and the use of statistical process control charts for determining optimal portfolio revisions (Golosnoy and Okhrin (2009), MacLean et al. (2006)). Both approaches require threshold values to trigger the revision process, however, rendering the results highly sensitive to the chosen threshold. Furthermore, all of the approaches mentioned thus far neglect the 
impact of estimation errors and any corresponding in-sample overoptimism on the revision decision.

Our paper contributes to the existing literature in three ways. First, our approach represents a new method to overcoming the problem of optimal portfolio revision by focusing on the (consistently estimated) expected out-of-sample performance of the revised portfolio, which we regard as the natural objective for revision. Accordingly, our approach takes into account the problem of estimation errors and in-sample overoptimism.

Second, we derive our estimator by optimizing expected out-of-sample portfolio performance. This represents an exact solution to the revision problem, rather than a heuristic one. By focusing on the expected out-of-sample performance of the revised portfolio, our approach is easily scalable and computationally inexpensive, even for large investment universes.

Third, our estimator gives a purely data-driven solution to the portfolio revision problem. It requires neither calibration nor a threshold value. It is thus easily implementable, and of interest for practical applications.

The remainder of this paper is organized as follows. Section 2 describes our framework for optimal portfolio revision, reviews the various minimum-variance portfolio strategies that we use to evaluate the profitability of our policy, and outlines our performance evaluation procedure. Section 3 discusses our proposed revision policy using simulated data, while section 4 explores its performance on empirical datasets. Section 5 gives our conclusions. 


\section{Methodology}

\subsection{Optimal Portfolio Revision Policy}

Throughout this paper, we consider the typical myopic minimum-variance portfolio optimization problem. The investor's objective is to minimize $w^{\prime} \Sigma w$, where $w$ denotes a column vector of optimal portfolio weights, and $\Sigma$ represents the population covariance matrix. Because $\Sigma$ is not observable, we use an estimate based on sample information, denoted by $S$. The sample estimate of the portfolio variance is thus $\hat{w}^{\prime} S \hat{w}$, where $\hat{w}$ represents the sample estimate of $w$.

In the traditional rolling sample approach to portfolio optimization, investors determine $\hat{w}_{t}$ at the end of each period $t$ using the sample information from the previous $\tau$ months. The portfolio weights are held constant over the consecutive period, $t+1$. At the end of $t+1$, the investor receives the corresponding out-of-sample return $\hat{w}_{t}^{\prime} R_{t+1}$, where $R_{t+1}$ denotes the cross-section of excess returns in period $t+1$. The investor then determines the new optimal portfolio weights, $\hat{w}_{t+1}$, conditional on the sample information of the new estimation period, which ranges from $t+2-\tau$ to $t+1$. Finally, investors completely revise their portfolio weights to the new portfolio weights, $\hat{w}_{t+1}$, since the old portfolio weights, $\hat{w}_{t}$, are no longer efficient conditional on the new in-sample information. This procedure is then repeated over the entire sample period.

However, Kan and Zhou (2007), Siegel and Woodgate (2007), and Basak et al. (2009) show that the in-sample variance of a portfolio is not a consistent estimator of expected out-of-sample variance, which intuitively appears to be the quantity of interest when investors revise their portfolios.

Basak et al. (2009) demonstrate that the in-sample variance of a portfolio suffers from overoptimism and systematically underestimates out-of-sample variance. Hence, because the new in-sample optimal portfolio weights do not necessarily yield a lower expected out-of-sample variance, investors may not be better off revising their weights 
from the previous period to the current period. Contrasting, it may be more advantageous to hold a combination of both weight estimates, because this could yield diversification benefits if the expected out-of-sample variances are not perfectly correlated. Moreover, holding the weights of the previous period constant may even yield a lower expected out-of-sample variance than shifting them toward the new ones.

Aside from the expected out-of-sample variance, managing the turnover resulting from a portfolio revision is crucial for the practical implementation of a portfolio strategy. Typically, higher turnover hampers implementation, and can adversely affect portfolio performance in the presence of trading costs. Hence, practitioners will generally prefer less frequent revisions of portfolio weights due to a decrease in turnover.

\subsubsection{Portfolio revision in a two-period setting}

For the sake of simplicity, we first describe our portfolio revision policy in a twoperiod setting. Our objective is to minimize the expected out-of-sample portfolio variance, $\operatorname{Var}\left(\hat{w}_{t}^{R e v^{\prime}} R_{t+1}\right)$, where $\hat{w}_{t}^{R e v}$ is the convex combination of the estimated portfolio weights of the current and previous period, denoted by $\hat{w}_{t}$ and $\hat{w}_{t-1}$, respectively. The portfolio weights of our proposed revision policy in period $t$ are obtained by $\hat{w}_{t}^{R e v}=\alpha \hat{w}_{t-1}+(1-\alpha) \hat{w}_{t}$, where $\alpha$ is the fraction of wealth that the investor keeps in the portfolio weights from the previous period, $\hat{w}_{t-1}$. We can express the quantity of interest (the expected out-of-sample portfolio variance), as follows:

$$
\min _{\alpha} \operatorname{Var}\left(\hat{w}_{t}^{R e v^{\prime}} R_{t+1}\right)=\operatorname{Var}\left(\alpha \hat{w}_{t-1}^{\prime} R_{t+1}+(1-\alpha) \hat{w}_{t}^{\prime} R_{t+1}\right) .
$$


The first and second derivatives of $\operatorname{Var}\left(\hat{w}_{t}^{R e v^{\prime}} R_{t+1}\right)$ yield:

$$
\begin{aligned}
\operatorname{Var}^{\prime}\left(\hat{w}_{t}^{R e v^{\prime}} R_{t+1}\right)= & \alpha \operatorname{Var}\left(\hat{w}_{t-1}^{\prime} R_{t+1}-\hat{w}_{t}^{\prime} R_{t+1}\right)-\operatorname{Var}\left(\hat{w}_{t}^{\prime} R_{t+1}\right) \\
& +\operatorname{Cov}\left(\hat{w}_{t-1}^{\prime} R_{t+1}, \hat{w}_{t}^{\prime} R_{t+1}\right), \\
\operatorname{Var}^{\prime \prime}\left(\hat{w}_{t}^{R e v^{\prime}} R_{t+1}\right)= & \operatorname{Var}\left(\hat{w}_{t-1}^{\prime} R_{t+1}-\hat{w}_{t}^{\prime} R_{t+1}\right) .
\end{aligned}
$$

Setting $\operatorname{Var}^{\prime}\left(\hat{w}_{t}^{R e v^{\prime}} R_{t+1}\right)=0$ and solving for $\alpha$ yields the optimal combination intensity, $\alpha^{*}$, which minimizes the expected out-of-sample portfolio variance as follows:

$$
\alpha^{*}=\frac{\operatorname{Var}\left(\hat{w}_{t}^{\prime} R_{t+1}\right)-\operatorname{Cov}\left(\hat{w}_{t-1}^{\prime} R_{t+1}, \hat{w}_{t}^{\prime} R_{t+1}\right)}{\operatorname{Var}\left(\hat{w}_{t-1}^{\prime} R_{t+1}-\hat{w}_{t}^{\prime} R_{t+1}\right)} .
$$

Because (3) is positive everywhere, the expression in (4) is verified as a global minimum for the expected out-of-sample variance. However, note that $\alpha^{*}$ is not a bona fide estimator, since it depends on the unobservable quantities $\operatorname{Var}\left(\hat{w}_{t}^{\prime} R_{t+1}\right), \operatorname{Cov}\left(\hat{w}_{t-1}^{\prime} R_{t+1}, \hat{w}_{t}^{\prime} R_{t+1}\right)$, and $\operatorname{Var}\left(\hat{w}_{t-1}^{\prime} R_{t+1}-\hat{w}_{t}^{\prime} R_{t+1}\right)$. Hence, we need to estimate the quantities in (4) consistently.

To do this, we use Basak et al.'s (2009) Jackknife-type estimator of the expected out-of-sample portfolio return moments. Following this methodology, we first drop the $i$-th observation from the in-sample period and compute the resulting covariance matrix based on the remaining $\tau-1$ returns, denoted by $S_{t, i}$. Next, we determine the corresponding optimal portfolio weights, denoted by $\hat{w}_{t, i}$. Finally, we compute the Jackknife return, $R_{t, i}^{J K}$, that would have been achieved from holding $\hat{w}_{t, i}$ in period $i$, given by $\hat{w}_{t, i}^{\prime} R_{i}$. Repeating the aforementioned process for all $i=\{1, . ., \tau\}$ leaves us with $\tau$ Jackknife returns $\left\{R_{t, 1}^{J K}, R_{t, 2}^{J K}, \cdots, R_{t, \tau}^{J K}\right\}$

Based on the underlying Jackknife assumption that returns on risky assets are identically and independently distributed over time, Basak et al. (2009) show that the distribution of $R_{t, i}^{J K}$ follows the same distribution as $\hat{w}_{t}^{\prime} R_{t+1}$. The sample variance based on the $\tau$ Jackknife returns thus represents a natural estimator of the out-of-sample variance, 
$\operatorname{Var}\left(\hat{w}_{t}^{\prime} R_{t+1}\right)$, denoted by:2

$$
\begin{array}{r}
\widehat{V A R}^{J K}\left(\hat{w}_{t}^{\prime} R_{t+1}\right)=\frac{1}{\tau} \sum_{i=1}^{\tau}\left(R_{t, i}^{J K}-\mu_{\hat{w}_{t}}\right)^{2}, \text { with } \\
\mu_{\hat{w}_{t}}=\frac{1}{\tau} \sum_{i=1}^{\tau} R_{t, i}^{J K} .
\end{array}
$$

Note that when we estimate the remaining quantities in Equation (4), $\operatorname{Cov}\left(\hat{w}_{t-1}^{\prime} R_{t+1}, \hat{w}_{t}^{\prime} R_{t+1}\right)$ and $\operatorname{Var}\left(\hat{w}_{t-1}^{\prime} R_{t+1}-\hat{w}_{t}^{\prime} R_{t+1}\right)$, we only find $\tau-1$ overlapping Jackknife returns available for computation.

This is attributable to the use of the rolling sample procedure. The intuition of this procedure is that only the last $\tau$ observations are relevant for determining the quantity of interest. We thus need to compute $\operatorname{Cov}\left(\hat{w}_{t-1}^{\prime} R_{t+1}, \hat{w}_{t}^{\prime} R_{t+1}\right)$ and $\operatorname{Var}\left(\hat{w}_{t-1}^{\prime} R_{t+1}-\hat{w}_{t}^{\prime} R_{t+1}\right)$ based on the same observations (the most recent $\tau$ returns).

In period $t$ the Jackknife return for $\hat{w}_{t-1}$ is missing in the last period of the sample used to estimate $\hat{w}_{t}$. To overcome this problem, we compute the out-of-sample return for $\hat{w}_{t-1}$ for the missing observation, which is $\hat{w}_{t-1}^{\prime} R_{t}$. From this, we obtain a vector of approximate Jackknife returns $s^{3}$ for $\hat{w}_{t-1}$, consisting of $\left\{\hat{w}_{t-1}^{\prime} R_{t}, R_{t-1,1}^{J K}, R_{t-1,2}^{J K}, \cdots, R_{t-1, \tau-1}^{J K}\right\}$. Because the sequence of all Jackknife returns for $\hat{w}_{t-1}$ has the same distribution as $\hat{w}_{t-1}^{\prime} R_{t}$, using it does not alter the distribution. It further enables us to obtain consistent estimates of $\operatorname{Cov}\left(\hat{w}_{t-1}^{\prime} R_{t+1}, \hat{w}_{t}^{\prime} R_{t+1}\right)$ and $\operatorname{Var}\left(\hat{w}_{t-1}^{\prime} R_{t+1}-\hat{w}_{t}^{\prime} R_{t+1}\right)$ based on the last $\tau$ observations. We can thus estimate $\operatorname{Cov}\left(\hat{w}_{t-1}^{\prime} R_{t+1}, \hat{w}_{t}^{\prime} R_{t+1}\right)$ as:

$$
\begin{gathered}
\widehat{\operatorname{Cov}}^{J K}\left(\hat{w}_{t-1}^{\prime} R_{t+1}, \hat{w}_{t}^{\prime} R_{t+1}\right)=\frac{1}{\tau}\left(\left(\hat{w}_{t-1}^{\prime} R_{1}-\mu_{\hat{w}_{t-1}}\right)\left(R_{t, 1}^{J K}-\mu_{\hat{w}_{t}}\right)\right)+ \\
\frac{1}{\tau} \sum_{i=2}^{\tau}\left(R_{t-1, i}^{J K}-\mu_{\hat{w}_{t-1}}\right)\left(R_{t, i}^{J K}-\mu_{\hat{w}_{t}}\right), \text { with } \\
\mu_{\hat{w}_{t-1}}=\frac{1}{\tau}\left(\hat{w}_{t-1}^{\prime} R_{1}+\sum_{i=2}^{\tau} R_{t-1, i}^{J K}\right) \text { and } \mu_{\hat{w}_{t}} \text { as defined in (6), }
\end{gathered}
$$

\footnotetext{
${ }^{2}$ See Basak et al. (2009) for further discussion of this point.

${ }^{3}$ The term "approximate Jackknife returns" accounts for the mixture of out-of-sample returns and Jackknife returns in the vector.
} 
and $\operatorname{Var}\left(\hat{w}_{t-1}^{\prime} R_{t+1}-\hat{w}_{t}^{\prime} R_{t+1}\right)$ as:

$$
\begin{gathered}
\widehat{\operatorname{Var}}^{J K}\left(\hat{w}_{t-1}^{\prime} R_{t+1}-\hat{w}_{t}^{\prime} R_{t+1}\right)=\frac{1}{\tau}\left(\left(\hat{w}_{t-1}^{\prime} R_{1}-R_{t, 1}^{J K}\right)-\mu_{\hat{w}_{t-1}-\hat{w}_{t}}\right)^{2}+ \\
\frac{1}{\tau} \sum_{i=2}^{\tau}\left(\left(R_{t-1, i}^{J K}-R_{t, i}^{J K}\right)-\mu_{\hat{w}_{t-1}-\hat{w}_{t}}\right)^{2}, \text { with } \\
\mu_{\hat{w}_{t-1}-\hat{w}_{t}}=\frac{1}{\tau}\left(\left(\hat{w}_{t-1}^{\prime} R_{1}-R_{t, 1}^{J K}\right)+\sum_{i=2}^{\tau} R_{t-1, i}^{J K}-R_{t, i}^{J K},\right),
\end{gathered}
$$

which finally allows us to estimate $\alpha^{*}$ as:

$$
\hat{a}^{*}=\frac{\widehat{\operatorname{Var}}^{J K}\left(\hat{w}_{t}^{\prime} R_{t+1}\right)-\widehat{\operatorname{Cov}}^{J K}\left(\hat{w}_{t-1}^{\prime} R_{t+1}, \hat{w}_{t}^{\prime} R_{t+1}\right)}{\widehat{\operatorname{Var}}^{J K}\left(\hat{w}_{t-1}^{\prime} R_{t+1}-\hat{w}_{t}^{\prime} R_{t+1}\right)} .
$$

Naturally, $\hat{a}^{*}$ may lie outside the desired range between 0 (the portfolio is totally revised to the new portfolio weights) and 1 (the portfolio is not revised at all). To ensure that $\hat{a}^{*}$ lies within the desired interval, we cut off values that lie outside the $[0,1]$ interval, so that:

$$
\hat{a}=\max \left[0, \min \left(1, \hat{a}^{*}\right)\right] .
$$

\subsubsection{Portfolio revision in a multi-period setting}

It is straightforward to extend the revision scheme of the two-period case to a multi-period setting. In a multi-period setting, the weights of the previous period are already a convex combination of those from the preceding $t-1$ periods. However, two minor adjustments are necessary. First, we must change the notation of the old portfolio weights from the

previous period from $\hat{w}_{t-1}$ to $\hat{w}_{t-1}^{R e v}$ to account for the fact that $\hat{w}_{t-1}^{R e v}$ may not fully be determined by $\hat{w}_{t-1}$. In other words, the revised portfolio weights of the last period have not been or have only been partially revised to the portfolio weight estimate $\hat{w}_{t-1}$.

Second, we must track the solution to the out-of-sample variance minimization problem in (1) over time, to determine both, the share of less recent portfolio weight estimates 
of $\hat{w}_{t-1}^{R e v}$ and the corresponding determination of the expected out-of-sample variance. We can thus describe investors' revision decisions in each period $t$ by the following minimization problem:

$$
\min _{\alpha_{t}} \operatorname{Var}\left(\hat{w}_{t}^{R e v^{\prime}} R_{t+1}\right)=\operatorname{Var}\left(\alpha_{t} \hat{w}_{t-1}^{R e v^{\prime}} R_{t+1}+\left(1-\alpha_{t}\right) \hat{w}_{t}^{\prime} R_{t+1}\right) .
$$

Similarly to the two-period case, this solves as:

$$
\alpha_{t}^{*}=\frac{\operatorname{Var}\left(\hat{w}_{t}^{\prime} R_{t+1}\right)-\operatorname{Cov}\left(\hat{w}_{t-1}^{R e v^{\prime}} R_{t+1}, \hat{w}_{t}^{\prime} R_{t+1}\right)}{\operatorname{Var}\left(\hat{w}_{t-1}^{R e v^{\prime}} R_{t+1}-\hat{w}_{t}^{\prime} R_{t+1}\right)} .
$$

Again, we face the problem that $\alpha_{t}^{*}$ is not a bona fide estimator. Accordingly, we need to estimate $\operatorname{Var}\left(\hat{w}_{t}^{\prime} R_{t+1}\right), \operatorname{Cov}\left(\hat{w}_{t-1}^{R e v^{\prime}} R_{t+1}, \hat{w}_{t}^{\prime} R_{t+1}\right)$, and $\operatorname{Var}\left(\hat{w}_{t-1}^{R e v^{\prime}} R_{t+1}-\hat{w}_{t}^{\prime} R_{t+1}\right)$. Because $\operatorname{Var}\left(\hat{w}_{t}^{\prime} R_{t+1}\right)$ does not differ from the two-period case, we can estimate it by using the estimator in (5).

Estimating $\operatorname{Cov}\left(\hat{w}_{t-1}^{R e v^{\prime}} R_{t+1}, \hat{w}_{t}^{\prime} R_{t+1}\right)$ and $\operatorname{Var}\left(\hat{w}_{t-1}^{R e v^{\prime}} R_{t+1}-\hat{w}_{t}^{\prime} R_{t+1}\right)$, however, is more complex. Because both quantities depend on $\hat{w}_{t-1}^{R e v}$ it may be a convex combination of $\left\{\hat{w}_{t-1}, \hat{w}_{t-2}, \ldots, \hat{w}_{t-n}\right\}$. We thus need to compute the expected out-of-sample variance for $\hat{w}_{t-1}^{R e v^{\prime}}$ by taking all $n$ portfolio weights into account. ${ }^{4}$

To illustrate this in practice, let $r_{j, t}$ denote the vector of approximate Jackknife returns corresponding to $\hat{w}_{t}$, where $j$ denotes the period for which the portfolio weights and the corresponding Jackknife returns $\left\{R_{j, 1}^{J K}, R_{j, 2}^{J K}, \cdots, R_{j, \tau}^{J K}\right\}$ were initially calculated. Whenever $\alpha_{t}$ takes a value of 0 , the following procedure applies to the consecutive periods: we obtain return vector $r_{t, t}$, consisting of $\tau$ Jackknife returns $\left\{R_{t, 1}^{J K}, R_{t, 2}^{J K}, \cdots, R_{t, \tau}^{J K}\right\}$ in the first period, $t$. In the following period, $t+1$, we obtain the new return vector, $r_{t+1, t+1}$, which again consists of $\tau$ Jackknife returns $\left\{R_{t+1,1}^{J K}, R_{t+1,2}^{J K}, \cdots, R_{t+1, \tau}^{J K}\right\}$. The

\footnotetext{
${ }^{4}$ Note that the number of portfolio weights, $n$, contained in $\hat{w}_{t-1}^{R e v}$ depends on the historical values of $\left\{\alpha_{t-1}, \alpha_{t-2}, \cdots \alpha_{t-n}\right\}$. As long as none of the $n$ alphas is equal to zero, $\hat{w}_{t-1}^{R e v}$ will be a convex combination of all portfolio weights determined over the $n$ periods. In contrast, as soon as any alpha equals zero, the portfolio weights are completely revised to the new, in-sample optimal weights, which render the old weights and their corresponding approximate Jackknife returns obsolete.
} 
previous return vector, $r_{t, t}$, then becomes $r_{t, t+1}$ in $t+1$, indicating that it comprises $\tau-1$ Jackknife returns corresponding to portfolio $\hat{w}_{t}$ and one out-of sample return $\left\{\hat{w}_{t}^{\prime} R_{t+1}, R_{t, 1}^{J K}, R_{t, 2}^{J K}, \cdots, R_{t, \tau-1}^{J K}\right\}$. After the revision decision in $t+1$, we determine the approximate Jackknife return vector of the revised portfolio in $t+1, r_{t+1, t+1}^{R e v}$, as follows:

$$
r_{t+1, t+1}^{R e v}=\alpha_{1} r_{t, t+1}+\left(1-\alpha_{1}\right) r_{t+1, t+1}
$$

Consequently, the entries in the vector of approximate Jackknife returns $r_{t+1, t+1}^{R e v}$ are given by $\left\{\alpha_{1} \hat{w}_{t}^{\prime} R_{t+1}+\left(1-\alpha_{1}\right) R_{t+1,1}^{J K}, \alpha_{1} R_{t, 1}^{J K}+\left(1-\alpha_{1}\right) R_{t+1,2}^{J K}, \cdots, \alpha_{1} R_{t, \tau-1}^{J K}+\left(1-\alpha_{1}\right) R_{t+1, \tau}^{J K}\right\}$. In the next period, the same procedure applies as follows:

$$
\begin{aligned}
r_{t+2, t+2}^{R e v} & =\alpha_{2}\left[\alpha_{1} r_{t, t+2}+\left(1-\alpha_{1}\right) r_{t+1, t+2}\right]+\left(1-\alpha_{2}\right) r_{t+2, t+2} \\
& =\alpha_{2} r_{t+1, t+2}^{R e v}+\left(1-\alpha_{2}\right) r_{t+2, t+2}
\end{aligned}
$$

It is obvious that, unless the $n$-th $\alpha_{t}$ takes a value of zero, the vector of approximate Jackknife returns corresponding to $w_{t}$ is determined by:

$$
r_{t+n, t+n}^{R e v}=\alpha_{n} r_{t+n-1, t+n}^{R e v}+\left(1-\alpha_{n}\right) r_{t+n, t+n}
$$

Accordingly, let $r_{t-1, t, i}^{R e v}$ and $r_{t, t, i}$ denote the $i$-th element of the vector $r_{t-1, t}^{R e v}$ and $r_{t, t}$, respectively. Following the intuition of the revision scheme described above, we can then estimate $\operatorname{Cov}\left(\hat{w}_{t-1}^{R e v^{\prime}} R_{t+1}, \hat{w}_{t}^{\prime} R_{t+1}\right)$ as:

$$
\widehat{\operatorname{Cov}}^{J K}\left(\hat{w}_{t-1}^{R e v^{\prime}} R_{t+1}, \hat{w}_{t}^{\prime} R_{t+1}\right)=\frac{1}{\tau} \sum_{i=1}^{\tau}\left(r_{t-1, t, i}^{R e v}-\frac{1}{\tau} \sum_{i=1}^{\tau} r_{t-1, t, i}^{R e v}\right)\left(r_{t, t, i}-\frac{1}{\tau} \sum_{i=1}^{\tau} r_{t, t, i}\right)
$$

and $\operatorname{Var}\left(\hat{w}_{t-1}^{R e v^{\prime}} R_{t+1}-\hat{w}_{t}^{\prime} R_{t+1}\right)$ as:

$$
\widehat{\operatorname{Var}}^{J K}\left(\hat{w}_{t-1}^{R e v^{\prime}} R_{t+1}-\hat{w}_{t}^{\prime} R_{t+1}\right)=\frac{1}{\tau} \sum_{i=1}^{\tau}\left(\left(r_{t-1, t, i}^{R e v}-r_{t, t, i}\right)-\frac{1}{\tau} \sum_{i=1}^{\tau}\left(r_{t-1, t, i}^{R e v}-r_{t, t, i}\right)\right)^{2}
$$


which finally allows us to estimate $\alpha^{*}$ as:

$$
\hat{a}^{*}=\frac{\widehat{\operatorname{Var}}^{J K}\left(\hat{w}_{t}^{\prime} R_{t+1}\right)-\widehat{\operatorname{Cov}}^{J K}\left(\hat{w}_{t-1}^{R e v^{\prime}} R_{t+1}, \hat{w}_{t}^{\prime} R_{t+1}\right)}{\widehat{\operatorname{Var}}^{J K}\left(\hat{w}_{t-1}^{R e v^{\prime}} R_{t+1}-\hat{w}_{t}^{\prime} R_{t+1}\right)} .
$$

Analogously to the two-period case, we trim the estimator $\hat{a}^{*}$ according to Equation 10 in order to ensure that the values lie inside the $[0,1]$ interval.

\subsection{Description of Evaluated Portfolios}

In this section, we briefly describe the minimum-variance portfolio strategies used to evaluate our proposed revision policy. The difference between the various portfolio strategies is attributable to the different estimators of the variance-covariance matrix. All portfolio weights are computed by solving the following optimization problem:

$$
\begin{aligned}
& \min _{\hat{w}} \hat{w}^{\prime} S \hat{w} \\
\text { s.t. } & \hat{w}^{\prime} 1_{N}=1,
\end{aligned}
$$

where $1_{N}$ denotes a vector of ones of appropriate size. The well-known solution to this minimization problem is given by:

$$
\hat{w}=S^{-1} 1_{N}\left(1_{N}^{\prime} S^{-1} 1_{N}\right)^{-1}
$$

which is the sample estimate of the global minimum-variance portfolio weights. For our analysis, we focus only on the most prominent covariance matrix estimators and the corresponding minimum-variance portfolios from the portfolio selection literature. Table 1 provides an overview of the portfolios.

[INSERT TABLE 11 HERE] 
We divide our sample of minimum-variance portfolios into two groups: one based on a single covariance matrix estimate, and the other based on a combination of two covariance matrix estimators. In the first group, we consider three covariance matrix estimators. We evaluate the minimum-variance portfolio based on the sample covariance matrix (SAMPLE), as follows:

$$
S_{S A M P L E}=\frac{1}{\tau-1} X X^{\prime}
$$

where $X$ denotes a $\tau \times N$ matrix of de-meaned returns. Next, we consider the suitability of our revision policy for the short sale-constrained minimum-variance portfolio (MINC). To compute the optimal weights, the optimization problem in equations $(16)-(17)$ is extended by adding a weight constraint:

$$
\hat{w}_{i} \geq 0, i=1,2, \ldots, N \text {. }
$$

Jagannathan and Ma (2003) show that the portfolio weights of the short sale constrained minimum-variance portfolio correspond to those of the global minimum-variance portfolio using the following covariance matrix estimator:

$$
S_{S C}=S_{S A M P L E}-\left(\lambda 1^{\prime}+1 \lambda^{\prime}\right)
$$

where $\lambda=\left\{\lambda_{1}, \lambda_{2}, \ldots, \lambda_{N}\right\}$ denotes a vector of Lagrangian multipliers for the nonnegativity constraints in 19 .

Third, we assess our revision policy by using a minimum-variance portfolio based on the one-factor covariance matrix (1F). For this analysis, we use Sharpe's (1967) singleindex model which assumes that the return of stock $i$ at time $t, r_{i, t}$, can be described by:

$$
r_{i, t}=\alpha_{i}+\beta_{i} r_{M, t}+\delta_{i, t},
$$


where $\alpha_{i}$ is the mispricing of stock $i, \beta_{i}$ is the beta factor of stock $i, r_{M, t}$ is the excess return of the market over the risk-free asset at time $t$, and $\delta_{i, t}$ is the residual variance of stock $i$ at time $t$. The covariance matrix estimate implied by this model is thus:

$$
S_{1 F}=\hat{\sigma}_{M}^{2} \hat{\beta} \hat{\beta}^{\prime}+\hat{\Delta}
$$

where $\hat{\sigma}_{M}, \hat{\beta}$, and $\hat{\Delta}$ are the estimates of market variance, beta factors, and a diagonal matrix with the residual variances $\hat{\delta}_{i, t}$, respectively. $:^{5}$

The second group of minimum-variance portfolios is comprised of the three shrinkage estimators proposed by Ledoit and Wolf (2003, 2004a, 2004b). All the shrinkage estimators of the covariance matrix considered here take the following form:

$$
S_{L W}=\phi \hat{F}+(1-\phi) S_{S A M P L E}
$$

Obviously, $S_{L W}$ is a convex combination of the sample covariance matrix and a shrinkage target $\hat{F}$, with the shrinkage intensity, $\phi$, taking values between 0 and 1 . The optimal shrinkage intensity is determined by minimizing the quadratic loss of $S_{L W}{ }^{6}$ Following the authors, we consider three different candidates for $\hat{F}$ : the single-factor model covariance matrix (LW1F) (Ledoit and Wolf (2003)), a multiple of the identity matrix (LWID) (Ledoit and Wolf (2004a)), and the constant correlation model (LWCC) (Ledoit and Wolf (2004b)).

\footnotetext{
${ }^{5}$ Following the results of Chan et al. (1999), we do not include any other factor models here except for the single index model, because additional factors have not been shown to improve portfolio performance.

${ }^{6}$ We refer the interested reader to the papers by Ledoit and Wolf (2003, 2004a, 2004b) for a derivation of the optimal shrinkage intensity and an in-depth discussion of the estimators. See also a recent paper of Kourtis et al. (2012), who apply the shrinkage methodology directly to the inverse covariance matrix using non-parametric techniques.
} 


\subsection{Performance Evaluation Method}

In order to evaluate the profitability of our proposed revision policy, we benchmark the performance of each portfolio strategy based on our policy relative to its performance based on other revision frequencies. We use benchmarks of one, six, and twelve months, 7 and assess profitability for each portfolio strategy $i$ using four performance measures: portfolio variance $\left(\hat{\sigma}^{2}\right)$, the Sharpe ratio $(\widehat{S R})$, the certainty equivalent $(\widehat{C E Q})$ return, and the average portfolio turnover $(T R N)$ :

$$
\begin{array}{r}
\hat{\sigma}_{i}^{2}=\frac{1}{T-\tau-1} \sum_{t=\tau}^{T-1}\left(w_{i, t}^{\prime} r_{t+1}-\hat{\mu}_{i}\right)^{2} \\
\text { with } \hat{\mu}_{i}=\frac{1}{T-\tau} \sum_{t=\tau}^{T-1} w_{i, t}^{\prime} r_{t+1}, \\
\widehat{S R_{i}}=\frac{\hat{\mu}_{i}-r_{f}}{\hat{\sigma}_{i}}, \\
\widehat{C E Q_{i}}=\hat{\mu}_{i}-\frac{\gamma}{2} \hat{\sigma}_{i}^{2}, \\
T R N_{i}=\frac{1}{T-\tau-1} \sum_{t=\tau}^{T-1} \sum_{j=1}^{N}\left|w_{i, j, t+1}-w_{i, j, t+}\right|,
\end{array}
$$

where $w_{i, j, t}$ denotes the portfolio weight of strategy $i$ in asset $j$ at time $t, w_{i, j, t+}$ is the corresponding weight before rebalancing at time $t+1$, and $w_{i, j, t+1}$ the congruent weight after rebalancing at time $t+1$.

To measure the statistical significance of the differences in performance metrics between our proposed revision policy and the benchmark revision strategies, we use bootstrapping methods. We explicitly control for the impact of time series effects (e.g., autocorrelation, volatility clustering, and non-normality) in portfolio returns and turnover

\footnotetext{
${ }^{7}$ Our choice of benchmark revision frequencies is driven by the commonly employed revision frequencies in the contemporaneous portfolio choice literature. While more recent studies (e.g., DeMiguel et al. (2009a, 2009b) and Tu and Zhou (2011)) employ a monthly revision scheme, the contributions by Chan et al. (1999), Jagannathan and Ma (2003), as well as Ledoit and Wolf (2003), are based on a twelve month revision frequency. Besides the considered one, six, and twelve months revision frequencies, we compute all results for a quarterly revision frequency. Given the qualitative and quantitative similarity of the three and six months revision frequency, we do not report the results for the three months revision frequency. However, the results are available from the authors upon request.
} 
on the performance metrics. Accordingly, for each portfolio strategy, we test whether the performance metric difference between our policy and a fixed revision frequency is statistically different from zero.

We next describe our procedure for conducting hypothesis testing to capture the time series effects of turnovers 8 For monthly turnover, we use the circular block bootstrap of Politis and Romano (1992). We randomly draw a fixed number, $b$, of consecutive observations (i.e., blocks of turnovers from the original time series of monthly turnovers), which is wrapped into a circle. We repeat the random draw of turnover blocks $m$ times, so that $m b=T-\tau$, which equals the original size of the time series of monthly turnovers. By repeating this $K$ times, we obtain $K$ bootstrap samples, each comprised of a time series of $T-\tau$ monthly turnovers.

We can now assess whether the empirical difference in average monthly turnover between a particular portfolio strategy based on our revision policy, $O p t$, is significantly different from the same strategy using a fixed portfolio revision frequency $n$. In other words, $H_{0}: t r n_{O p t}-t r n_{n}=0$. We compute the corresponding two-sided $p$-value for the stated null hypothesis using remark 3.2 by Ledoit and Wolf $(2008)$ as follows: Let $d$ denote the absolute value of the empirical average turnover difference between our revision policy, Opt, and the considered benchmark portfolio revision frequencies $n$. Further, let $d_{k}^{B}$ denote the absolute value of the same difference based on the $k$-th bootstrap sample. We may then compute the $p$-value as:

$$
P V=\frac{\#\left\{d_{k}^{B}>d\right\}+1}{K+1} .
$$

For the purpose of performance hypothesis testing using the variance, we follow Ledoit and Wolf (2011) and employ a studentized circular block bootstrap. We compute the $p$-value for the null hypothesis that the variance difference between a particular portfolio

\footnotetext{
${ }^{8}$ The remaining approaches to bootstrap hypothesis testing follow the described procedure closely, and differ only with respect to the resampling of the test statistic. The procedure for the $p$-value computation is equivalent across all approaches.
} 
strategy based on our revision policy, Opt, and a benchmark revision frequency, $n$, is significantly different from zero: $H_{0}=\log \left(\hat{\sigma}_{O p t}^{2}\right)-\log \left(\hat{\sigma}_{n}^{2}\right)=0.9$

For hypothesis testing with the Sharpe ratio, we employ Ledoit and Wolf's (2008) studentized circular block bootstrap. Again, we report the two-sided $p$-value for the null hypothesis, that the Sharpe ratio for a portfolio strategy using our revision policy, Opt, is equal to that of the same strategy revised according to $n: H_{0}=\widehat{S R}_{O p t}-\widehat{S R}_{n}=0$.

Similarly to the inference from the Sharpe ratio, we apply a modified version of Ledoit and Wolf's (2008) studentized circular block bootstrap for hypothesis testing with the certainty equivalent (CEQ) return 10 We then obtain the two-sided $p$-value for the following null hypothesis: $H_{0}=\widehat{C E Q}_{O p t}-\widehat{C E Q}_{n}=0$. For all bootstraps, we use a block length of $b=5$ and base our reported $p$-values on $K=1,000$ bootstrap iterations.

\section{Simulation Study}

In this section we use simulated data to evaluate the performance of our portfolio revision policy based on i.i.d. data, i.e. under clinical conditions, where results are not influenced by time series characteristics of asset returns. Our simulation setup follows Tu and Zhou's (2011) approach and parameterization. Specifically, we simulate returns according to a three-factor model with mispricing, which takes the form:

$$
r_{i, t}=\beta_{i, 0}+\beta_{i, 1} r_{t, \text { Market }}+\beta_{i, 2} r_{t, \text { Size }}+\beta_{i, 3} r_{t, B / M}+\epsilon_{i, t}
$$

We model the factor returns to follow a multivariate normal distribution. The means and covariance matrix match the empirical values of monthly returns on the three Fama and French (1993) factors (market, size, and book-to-market) from July 1963 to August 2007.11

\footnotetext{
${ }^{9}$ The log-transformation follows Ledoit and Wolf $(2011)$ in order to obtain better finite-sample properties. ${ }^{10}$ The adjustments to the studentized block bootstrap of Ledoit and Wolf (2008) are outlined in Appendix A.1.

${ }^{11}$ The choice of time horizon corresponds to that used by Tu and Zhou (2011).
} 
The factor loadings $\beta_{i, 1}, \beta_{i, 2}$, and $\beta_{i, 3}$, and the mispricing factor, $\beta_{i, 0}$, are constant over the simulation and randomly paired. The market factor loadings are spread evenly between 0.9 and 1.2, the size factor loadings are spread between -0.3 and 1.4, the book-to-market factor loadings are spread between -0.5 and 0.9 , and the mispricing factor is between -0.02 and 0.02. Note further that the variance-covariance matrix of noise is set to be diagonal, with the elements on the main diagonal drawn from a uniform distribution with support $[0.1,0.3]$. This results in a cross-sectional average annual idiosyncratic volatility of $20 \%$. Using the simulation setup described above, we generate $T=10,960$ monthly returns for an investment universe of $N=25$ assets, and an estimation period of $\tau=$ $\{60,120,240,480,960\}$.

\section{[INSERT TABLE 2 HERE]}

Table 2 shows that our proposed revision policy leads to significantly lower out-ofsample variances than the one-, six-, and twelve-month benchmark revision frequencies for all portfolio strategies. Furthermore, we observe that the decrease in out-of-sample variances is highly significant across all estimation periods. While the reductions are sizable for the shorter 60- and 120-month periods, they become relatively smaller for larger estimation periods of 240, 480, and 960 months. Yet, we find that the decrease in the out-of-sample variance remains statistically significant even for the largest estimation period of 960 months. We thus conclude that concentrating on expected out-of-sample variance as a revision criterion helps to achieve a lower realized out-of-sample variance.

\section{[INSERT TABLE 3 HERE]}

Tables 3 and 4 report the risk-adjusted performance as measured by the Sharpe ratio and the CEQ return. For the shorter estimation periods, we generally do not find any significant difference between our revision policy and the benchmark revision frequencies, except for the SAMPLE and 1F minimum-variance portfolio strategies. We find significant 
increases in the CEQ returns for both strategies when compared to all benchmark revision frequencies with a 60 months estimation period.

For the 240-months estimation period, we again observe significantly higher CEQ returns for the SAMPLE, 1F, and LWID strategies compared to all benchmark revision frequencies. We also note that our revision policy yields significantly higher Sharpe ratios for the $1 \mathrm{~F}$ portfolio strategy vis-à-vis the considered benchmark revision frequencies. For the 480- and 960-month estimation periods, we find significant increases in the Sharpe ratio and CEQ return across most portfolio strategies.

\section{[INSERT TABLE 4 HERE]}

The average monthly turnovers of the various portfolio strategies are reported in Table 5. The results show that our proposed revision policy yields a dramatic decrease in turnover compared to the benchmark revision frequencies. The turnover reductions are statistically significant for all portfolio strategies and estimation period sizes. For example, for the 60 months period, we observe a turnover decrease of between $48 \%$ (1F) and $66 \%$ (SAMPLE) vis-à-vis the commonly used monthly revision frequency.

We find similar figures for the six- and twelve-month revision frequencies, although the reductions become smaller due to the lower turnover of these benchmarks. Specifically, we find that we can reduce portfolio turnover by between $33 \%$ (1F and LWCC) and $49 \%$ (SAMPLE) compared to the six-month revision frequency. For twelve-month revision frequencies, we note reductions ranging from $24 \%$ (LWCC and Min-C) to $39 \%$.

Extending the estimation period from 60 to 120 months is accompanied by a decrease in turnover reduction. Accordingly, we find that our revision policy now reduces turnover by between $32 \%(1 \mathrm{~F})$ and $52 \%$ (SAMPLE) compared to the monthly frequency; by between $24 \%(1 \mathrm{~F})$ and $38 \%$ (SAMPLE) compared to the six-month frequency; and between $20 \%(1 \mathrm{~F})$ and $31 \%$ (SAMPLE) compared to the twelve-month frequency. We ascribe the lower turnover reduction of our policy compared to the shorter estimation 
periods to the generally higher stability of portfolio weights over time when the estimation period is increased. We thus find that the turnover reduction of our policy decreases proportionately with estimation period length.

For the 960-month estimation period, we find that our revision policy results in a roughly $5 \%$ decrease in turnover across all portfolio strategies compared to the considered benchmark revision frequencies. However, similarly to the out-of-sample variances, we note that even these small differences are significant.

\section{[INSERT TABLE 5 HERE]}

In summary, we find that our portfolio revision policy significantly reduces the outof-sample variance and turnover of the minimum-variance portfolio strategies considered here. The observed turnover reductions do not come at the cost of lower risk-adjusted performance as measured by the Sharpe ratio or the CEQ return. On the contrary, we find small increases in the performance metrics, particularly for long estimation periods.

In the following section, we move on from the clinical conditions of our simulation study, and evaluate our revision policy using real datasets.

\section{Empirical Results}

\subsection{Data}

Table 6 provides an overview of the five empirical datasets we use for this part of our evaluation: six and twenty-five portfolios sorted by size and book-to-market; ten and forty-eight industry portfolios, representing the U.S. stock market over our sample period, July 1963 through December 2008. Our single-stock dataset is comprised of the 100 largest U.S. single stocks by average market capitalization over the sample period from July 1965 through December 2008. The construction of this dataset is similar to that used in Chan et al. (1999), DeMiguel et al. (2009b), and Jagannathan and Ma (2003). We select all 
NYSE-, AMEX-, and Nasdaq-listed stocks from the CRSP database that have no missing returns over the entire sample period. From this filtered subset, we select the 100 largest stocks by average market capitalization over our sample period.

\section{[INSERT TABLE 6 HERE]}

The first four datasets are available from Kenneth French's websit 12 . For the riskfree rate, we use the 30-day T-bill rate, which was also obtained from Kenneth French's website.

\subsection{Evaluation of the Optimal Revision Policy}

Table 7 gives the out-of-sample variances for the analyzed portfolio strategies, revised according to our optimal portfolio revision policy (Opt) and the three benchmark revision frequencies (one, six, and twelve months). The portfolio variances of the optimal revision policy are generally comparable to those of the fixed revision frequencies. Yet, we observe several significant deviations for all datasets and portfolio strategies. We can thus draw two general conclusions.

First, the size of the asset universe is positively related to the amount of significant differences found. For the STOCK dataset, the SAMPLE, and 1F strategies have significantly lower out-of-sample variances when revising them according to our policy instead of using one of the three fixed revision frequencies. We attribute this to a shrinkage-like effect of our policy. Unless alpha takes the value of 1 , the revised portfolio weights from our revision policy are always a weighted average of the new and old weights (which may themselves be a combination of less recent weights), shrinking the new portfolio weight estimate towards the old portfolio weights.

[INSERT TABLE 7 HERE]

12 http://mba.tuck.dartmouth.edu/pages/faculty/ken.french/data_library.html. 
Second, we observe more significant differences in out-of-sample variance between our policy and the extended fixed revision frequencies, especially for the annual frequency. For most datasets and portfolio strategies, shorter fixed revision frequencies yield lower portfolio variances than longer ones. This is not surprising, because weights can be adjusted more quickly to changing market dynamics, e.g., the changes in correlation structure of individual assets or changes in volatility.

\section{[INSERT TABLE 8 HERE]}

Tables 8 and 9 report the results for the Sharpe ratio and the CEQ return which we compute for a risk aversion coefficient of five. We observe only a few significant performance differences between the optimal revision policy and the fixed frequencies. For example, we find significantly higher Sharpe ratios on the 6FF dataset for LWCC, on the 48IND dataset for SAMPLE, and on the STOCK dataset for $1 \mathrm{~F}$ and SAMPLE. Except for the observed deviations, we find that the Sharpe ratios and CEQ returns for the fixed frequencies are comparable to those for our optimal revision policy.

\section{[INSERT TABLE 9 HERE]}

Finally, Table 10 reports the portfolio turnovers. In almost all cases, the turnover from our optimal revision policy is significantly lower than that for the corresponding strategies revised according to a fixed frequency. The largest turnover differences are observed when comparing our revision policy to the monthly revision frequency. For example, the turnover of SAMPLE is reduced by $71.3 \%$ for the STOCK dataset, and the turnover of Min-C is reduced by $77.6 \%$ for the $25 \mathrm{FF}$ dataset.

However, even when compared to the annual revision frequency, we find considerable turnover reductions. We note that the turnovers of the $1 \mathrm{~F}$ and SAMPLE strategies for the STOCK dataset are reduced by $36.3 \%$ and $41.7 \%$, respectively. The average reductions for the portfolio strategies across all datasets range between: 31.6 and 66.5\% (SAMPLE), 
$30.9 \%$ and $40.4 \%(1 \mathrm{~F}), 20.1 \%$ and $53.2 \%$ (LW1F), $15.2 \%$ and $47.7 \%$ (LWID), $24.4 \%$ and $50.1 \%$ (LWCC), and $26.7 \%$ and $62.9 \%$ (Min-C), depending on the respective benchmark revision frequency. These results indicate that average turnover reductions are largely comparable across portfolio strategies.

[INSERT TABLE 10 HERE]

\subsection{Discussion of the Optimal Revision Intensities}

Figure 1 shows the time series of the $\alpha$ coefficients, representing the fraction of wealth that the investor keeps in portfolio weights from previous periods, for the STOCK dataset. The $\alpha$ coefficient takes values between $82.3 \%$ (LWCC) and $93.5 \%$ on average, representing less frequent and intense portfolio revisions as implied by our optimal revision policy. The corresponding inertia in the portfolio weights of the various considered minimum-variance portfolio strategies is reflected in the empirically observed lower turnover of the respective portfolio strategies.

\section{[INSERT FIGURE 1 HERE]}

The dynamics of the $\alpha$ coefficients differ across the various minimum-variance portfolio strategies. For instance, we observe that the $1 \mathrm{~F}$ strategy exhibits the longest time frame without any revision, while the SAMPLE strategy is frequently, but only partially revised. Despite apparent differences in the $\alpha$ coefficients, we note increased portfolio revisions in times of financial market turmoil for all portfolio strategies. Specifically, we note that all portfolio strategies show increased revision intensities in 1981, 1991, 1997, and 2001, which coincides with the savings and loan crisis, the Japan crisis, the Asian crisis, and the burst of the technology bubble.

For the remaining datasets, we observe overall similar patterns with the $\alpha$ coefficient showing a less volatile behavior over time with a decreasing size of the investment universe. 


\subsection{Optimal Revision Policy in the Presence of Transaction Costs}

Transaction costs play a vital role in the practical implementation of portfolio strategies. Considering them in the context of our revision policy evaluation allows us to quantify the economic value of our policy, e.g., how much of the increase in portfolio performance is attributable to the turnover reduction of our portfolio revision policy.

Note that our portfolio revision policy does not capture trading costs when determining what percentage of wealth should be kept in the old portfolio weights. While we could easily account for transaction costs when determining our optimal revision intensity, we find that this does not yield a favorable out-of-sample performance. We attribute this to the trade-off between an estimation error-prone quantity (the expected out-of-sample performance), and an estimation error-free quantity (trading costs) ${ }^{13}$

In order to assess the performance of our policy in the presence of transaction costs, we compute the differences of the Sharpe ratios and CEQ returns between our optimal revision policy and the fixed revision frequencies for all datasets and portfolio strategies. Specifically, we apply one-way transaction costs of between 0 and 100 bps, because the 50-bps midpoint results in round-trip costs of $1 \%$. This is in line with recent literature on transaction costs and portfolio optimization ${ }^{14}$

We consider it positive when our optimal revision policy achieves better performance than fixed revision frequencies with transaction costs of 50 bps or lower. The lower the transaction costs that are required to outperform the fixed revision frequencies, the better

\footnotetext{
${ }^{13}$ Our observations are in line with DeMiguel et al. (2010), who consider trading costs directly in the objective function of a myopic mean-variance portfolio optimization problem.

${ }^{14}$ See, for example, DeMiguel et al. (2009b), Kirby and Ostdiek (2011), and Brandt et al. (2009). These papers use proportional one-way transaction costs of $0.5 \%$ over a time period similar to the one used here. Brandt et al. (2009) models proportional transaction costs in a more sophisticated way by treating them as a function of market value and time. This accounts for the fact that transaction costs are typically lower for stocks with large market capitalizations, and that transaction costs have declined over time. Brandt et al. (2009) note that other authors have also found average proportional one-way transaction costs of about $0.5 \%$, which further confirms that this number is an appropriate assumption (see, e.g., Keim and Madhavan (1997) and Hasbrouck (2009)).
} 
is the performance of our proposed optimal revision policy.

\section{[INSERT FIGURE 2 HERE]}

Figure 2 shows the Sharpe ratio differences for the STOCK dataset. ${ }^{15}$ All curves have a positive slope because our proposed revision policy generates consistently lower turnover compared to the fixed revision frequencies. Thus, the performance differences increase along with higher transaction costs.

In almost all cases, the curve of the Sharpe ratio differences is above the zero line (where the Sharpe ratio difference is zero) for zero transaction costs. This indicates higher performance even without transaction costs for SAMPLE, Min-C, and 1F revised according to our revision policy. For LW1F with a twelve-month revision frequency, the Sharpe ratio difference curve intersects the zero line before transaction costs of 10 bps. This implies that, for transaction costs higher than 10 bps, our revision policy obtains a higher Sharpe ratio. For LWID with one- and six-month revision frequencies, we observe the intersections at similarly low transaction costs.

In addition to these results, we also note significant Sharpe ratio differences (at the $10 \%$ level) at or below transaction costs of 50 bps for many portfolio strategies and revision frequencies. Significant results are indicated by the intersection of the lower confidence band with the zero line.

Figure 3 depicts the CEQ return differences for the STOCK dataset. Overall, the results are highly comparable to those for the Sharpe ratio differences. All CEQ difference curves have a positive slope, which again increases with higher revision frequencies. For SAMPLE, Min-C, 1F, and LW1F, the CEQ return difference curves lie always above the zero line, representing larger CEQ returns for our proposed revision policy at zero transaction costs.

\footnotetext{
${ }^{15}$ We only present the figures for the Sharpe ratio and CEQ return differences for the STOCK dataset, because it represents tradable capital market data. The figures for the remaining datasets are omitted for the sake of space, but are available from the authors upon request.
} 
As for the Sharpe ratio differences, the CEQ return differences are mostly significantly different from zero, with the strongest results achieved for the portfolio strategies and revision frequencies with the largest turnovers (i.e., SAMPLE and 1F) at a monthly revision frequency.

\section{[INSERT FIGURE 3 HERE]}

For the remaining datasets $(6 \mathrm{FF}, 25 \mathrm{FF}, 10 \mathrm{Ind}$, and 30Ind), we observe a similar pattern for Sharpe ratio and CEQ return differences. We find a positive slope for Sharpe ratio and CEQ return difference curves across all datasets and for all portfolio strategies. Furthermore, in most cases, our proposed revision policy either has a positive Sharpe ratio, or CEQ return differences already at transaction costs of zero, or intersections at the zero line at reasonable transaction costs of 50 bps or below for the difference curves.

In addition to evaluating the statistical significance, we also aim to highlight the economic significance of our results. We compare the difference of the CEQ returns between the portfolios revised according to our policy, and those revised according to a fixed revision frequency. The result is the maximum management fee an investor would be willing to switch to our revision policy. At transaction costs of 50 bps an investor with the STOCK investment universe would be willing to pay annual management fees of between 1380 and 2304bps (SAMPLE), 27 and 63bps (Min-C), 336 and 360bps (1F), 24 and 96 bps (LW1F), -10 and 72bps (LWID), 0 and 72bps basis points (LWCC), and 27 and 63bps (Min-C), depending on the benchmark revision frequency.

Considering the economic significance across all datasets and portfolio strategies, we find that, for transaction costs of $50 \mathrm{bps}$, an investor with a risk aversion of five would be willing to pay an annual management fee of 101 bps for the optimal revision strategy, compared to the fixed annual revision frequency. For the semiannual and monthly revision frequencies, the corresponding management fees are 112 and $168 \mathrm{bps}$, respectively. We conclude from these numbers, that our revision policy adds a meaningful value to investors following one of the considered benchmark revision frequencies. 


\section{Conclusion}

This paper develops a novel approach to portfolio revision based on the expected outof-sample performance of the underlying portfolio strategy. Contrary to the commonly applied fixed revision frequencies, which suggest portfolio revisions after predefined time periods, our policy determines if and how much of the previous portfolio weights need to be revised. Our policy is thus purely data-driven and requires no calibration or threshold to trigger the revision of the portfolio.

In particular, our revision policy builds on Basak et al.'s (2009) Jackknife procedure to estimate the expected out-of-sample portfolio moments. Based on expected out-ofsample performance estimates, we derive a closed-form solution for the optimal percentage of wealth that should be allocated to the new in-sample optimal portfolio weights.

Using both simulations and empirical datasets, we demonstrate that our proposed revision policy leads to a substantial reduction in portfolio turnover compared to all considered fixed revision frequencies (one, six, and twelve months). Furthermore, our simulation results show that portfolios revised according to our policy achieve significantly reduced out-of-sample variances. We also find that risk-adjusted performance, as measured by the Sharpe ratio and the CEQ return, is equal to or slightly above that of the portfolios revised according to fixed revision frequencies.

Our empirical results largely confirm the findings from our simulation study. We note that our policy yields sizable turnover reductions compared to fixed revision frequencies, in some cases as high as $77.6 \%$. On average, we observe turnover reductions of $44.9 \%$, $27.4 \%$, and $18.9 \%$ compared to the one-, six-, and twelve-month frequencies, respectively. At the same time, we find that out-of-sample portfolio performance in terms of Sharpe ratios and CEQ returns is similar to or even slightly better than that obtained from using fixed revision frequencies. Moreover, when accounting for transaction costs, we note that the substantial turnover reductions attributable to our revision policy translate in many cases into economically and statistically significant gains in terms of Sharpe ratios and 
CEQ returns. On average, we find that an investor with a risk aversion of five would be willing to pay $101 \mathrm{bps}$ for the optimal revision strategy compared to the fixed annual revision frequency. When compared to semiannual and monthly frequencies, the amounts are 112 and 168 basis points, respectively. Finally, the results are particularly pronounced for the datasets with larger asset universes, where the turnover is usually high. 


\section{A Appendix}

\section{A.1 The studentized circular block bootstrap for hypothesis testing with the certainty equivalent return}

The difference between two Sharpe ratios is given by $\Delta_{S R}=f\left(\mu_{1}, \mu_{2}, \sigma_{1}, \sigma_{2}\right)=\frac{\mu_{1}}{\sigma_{1}}-\frac{\mu_{2}}{\sigma_{2}}$. Following Ledoit and Wolf (2008), use uncentered moments, where the difference between two Sharpe ratios is denoted by $\Delta_{S R}=f(u)=f(a, b, c, d)=\frac{a}{\sqrt{c-a^{2}}}-\frac{b}{\sqrt{d-b^{2}}}$. Equivalently, the difference between two certainty equivalent returns is given by:

$$
\Delta_{C E Q}=f(v)=f(a, b, c, d, \gamma)=a-\frac{1}{2} \gamma\left(c-a^{2}\right)-b+\frac{1}{2} \gamma\left(d-b^{2}\right)
$$

where $\gamma$ denotes the risk aversion coefficient. Let $\Delta_{C E Q}=f(v)=f(a, b, c, d, \gamma)$ denote the sample counterpart of $\hat{\Delta}_{C E Q}=f(\hat{v})=f(\hat{a}, \hat{b}, \hat{c}, \hat{d}, \gamma)$. Following Equation (4) in Ledoit and Wolf (2008), we can define the standard error of $\hat{\Delta}_{C E Q}$ as:

$$
s\left(\hat{\Delta}_{C E Q}\right)=\sqrt{\frac{\nabla^{\prime} f(\hat{v}) \hat{\Psi} \nabla f(\hat{v})}{T}},
$$

where $\nabla^{\prime} f(\hat{v})$ denotes the gradient of the certainty equivalent difference based on sample data, and $\hat{\Psi}$ is a consistent estimator of $\Psi$, which is an unknown symmetric positive semidefinite matrix. In particular, the gradient of the certainty equivalent return difference is given by:

$$
\nabla^{\prime} f(\hat{a}, \hat{b}, \hat{c}, \hat{d}, \gamma)=\left(1+\gamma \hat{a},-1-\gamma \hat{b},-\frac{1}{2} \gamma, \frac{1}{2} \gamma, \frac{1}{2}\left(\hat{a}^{2}-\hat{c}+\hat{d}-\hat{b}^{2}\right)\right)
$$

whereas $\hat{\boldsymbol{\Psi}}$ is obtained via kernel estimation, using Andrews and Monahan's (1992) prewhitened QS kernel (as proposed by Ledoit and Wolf (2008)).

In contrast to the gradient of the Sharpe ratio difference (see Ledoit and Wolf (2008) p. 852), the gradient of the certainty equivalent difference has five entries, because the 
difference depends not only on the sample moments $\hat{a}, \hat{b}, \hat{c}$, and $\hat{d}$, but also on the risk aversion coefficient $\gamma$.

For the inference about the sample certainty equivalent return difference, the standard error for each bootstrap sample of Ledoit and Wolf's (2008) studentized circle block bootstrap is given by:

$$
s\left(\hat{\Delta}_{C E Q}^{*}\right)=\sqrt{\frac{\nabla^{\prime} f\left(\hat{v}^{*}\right) \hat{\Psi}^{*} \nabla f(\hat{v})^{*}}{T}}
$$

where $s\left(\hat{\Delta}_{C E Q}^{*}\right), f\left(\hat{v}^{*}\right)$, and $\hat{\Psi}^{*}$ denote the bootstrap sample counterparts of $s\left(\hat{\Delta}_{C E Q}\right), f(\hat{v})$, and $\hat{\Psi}$. Following Ledoit and Wolf 2008) p. 854, we can then define:

$$
y_{t}^{*}=\left(r_{t i}^{*}-\hat{\mu}_{i}^{*}, r_{t n}^{*}-\hat{\mu}_{n}^{*}, r_{t i}^{* 2}-\hat{\Gamma}_{i}^{*}, r_{t n}^{* 2}-\hat{\Gamma}_{n}^{*}, 0\right) \text { for } t=1, . ., T
$$

where the last entry in $y_{t}^{*}$ is zero, because the risk aversion coefficient is held constant. Having the new $y_{t}^{*}$, and letting $l$ denote the number of blocks necessary to construct a bootstrap sample, Ledoit and Wolf 2008, p. 854) give the definitions for $\zeta_{j}$ and $\hat{\Psi}^{*}$ :

$$
\zeta_{j}=\frac{1}{\sqrt{b}} \sum_{t=1}^{b} y_{(j-1) b+t}^{*} \text { for } t=1, . ., T \text { and } \quad \hat{\Psi}^{*}=\frac{1}{l} \sum_{j=1}^{l} \zeta_{j} \zeta j^{\prime}
$$




\section{References}

Andrews, D. W. K., And J. C. Monahan (1992): "Heteroskedasticity and Autocorrelation Consistent Covariance Matrix Estimation," Econometrica, 59, 817-858.

Arnott, R. D., And J. R. M. Lovell (1993): "Rebalancing: Why, When, How Often?," Journal of Investing, 2, 5-10.

Basak, G. K., R. Jagannathan, and T. Ma (2009): "Jackknife Estimator for Tracking Error Variance of Optimal Portfolios," Management Science, 55, 990-1002.

Best, M. J., And R. R. Grauer (1991): "On the Sensitivity of Mean-Variance-Efficient Portfolios to Changes in Asset Means: Some Analytical and Computational Results," Review of Financial Studies, 4, 315-342.

Black, F., and R. Litterman (1992): "Global Portfolio Optimization," Financial Analysts Journal, 48, 28-43.

Brandt, M. W., P. Santa-Clara, and R. Valkanov (2009): "Parametric Portfolio Policies: Exploiting Characteristics in the Cross-Section of Equity Returns," Review of Financial Studies, 22, 3411-3447.

Chan, L. K. C., J. Karceski, and J. Lakonishok (1999): "On Portfolio Optimization: Forecasting Covariances and Choosing the Risk Model," Review of Financial Studies, 12, 937-974.

Chen, A. H. Y., F. C. Chen, and S. Zionts (1971): "The Optimal Portfolio Revision Policy," Journal of Financial and Quantitative Analysis, 44, 51-61.

Chopra, V. K. (1993): "Improving Optimization," Journal of Investing, 8, 51-59.

Chopra, V. K., C. R. Hensel, and A. L. Turner (1993): "Massaging MeanVariance Inputs: Returns from Alternative Global Investment Strategies in the 1980s," Management Science, 39, 845-855.

Chopra, V. K., and W. T. Ziemba (1993): "The Effects of Errors in Means, Variances, and Covariances on Optimal Portfolio Choice," Journal of Portfolio Management, 4, $6-11$.

Demiguel, V., L. Garlappi, F. J. Nogales, and R. Uppal (2009a): "A Generalized Approach to Portfolio Optimization: Improving Performance by Constraining Portfolio Norms," Management Science, 55, 798-812. 
DeMiguel, V., L. Garlappi, and R. Uppal (2009b): "Optimal Versus Naive Diversification: How Inefficient is the 1/N Portfolio Strategy?," Review of Financial Studies, 22, 1915-1953.

DeMiguel, V., F. J. Nogales, and R. Uppal (2010): "Stock Return Serial Dependence and Out-of-Sample Portfolio Performance," Working Paper.

Donhue, C., And K. YIP (2003): "Optimal Portfolio Rebalancing with Transaction Costs," Journal of Portfolio Management, 29, 49-63.

Fama, E. F., And K. R. French (1993): "Common Risk Factors in the Returns on Stock and Bonds," Journal of Financial Economics, 33, 3-56.

Golosnoy, V., And Y. Okhrin (2009): "EWMA Control Charts for Monitoring Optimal Portfolio Weights," Sequential Analysis, 26, 195-224.

Green, R., and B. Hollifield (1992): "When Will Mean-Variance Efficient Portfolios Be Well Diversified?," Journal of Finance, 47, 1785-1809.

Hasbrouck, J. (2009): "Trading Costs and Returns for US Equities: Estimating Effective Costs from Daily Data," Journal of Finance, 64, 1445-1477.

Jagannathan, R., And T. Ma (2003): "Risk Reduction in Large Portfolios: Why Imposing the Wrong Constraints Helps," Journal of Finance, 58, 1651-1684.

Jobson, J. D., And B. Korkie (1981): "Putting Markowitz Theory to Work," Journal of Portfolio Management, 7, 70-74.

Kamin, J. H. (1975): "Optimal Portfolio Revision with a Proportional Transaction Cost," Management Science, 21, 1263-1271.

Kan, R., And G. Zhou (2007): "Optimal Portfolio Choice with Parameter Uncertainty," Journal of Financial and Quantitative Analysis, 42, 621-656.

Keim, D. B., And A. Madhavan (1997): "Transactions Costs and Investment Style: An Inter-Exchange Analysis of Institutional Equity Trades," Journal of Financial Economics, 46, 265-292.

Kirby, C., And B. Ostdiek (2011): "It is All in the Timing: Simple Active Portfolio Strategies that Outperform Naive Diversification," Journal of Financial and Quantitative Analysis, forthcoming. 
Kourtis, A., G. Dotsis, and R. N. Markellos (2012): "Parameter Uncertainty in Portfolio Selection: Shrinking the Inverse Covariance Matrix," Journal of Banking and Finance, 36 (9), 2522-2531.

Kritzman, M., S. Myrgren, and S. Page (2009): "Optimal Rebalancing: A Scalable Solution," Journal of Investment Management, 7, 9-19.

Ledoit, O., And M. Wolf (2003): "Improved Estimation of the Covariance Matrix of Stock Returns with an Application to Portfolio Selection," Journal of Empirical Finance, 10, 603-621.

(2004a): "A Well-Conditioned Estimator for Large-Dimensional Covariance Matrices," Journal of Multivariate Analysis, 88, 365-411.

- (2004b): "Honey, I Shrunk the Sample Covariance Matrix," Journal of Portfolio Management, 30, 110-119.

_ (2008): "Robust Performance Hypothesis Testing with the Sharpe Ratio," Journal of Empirical Finance, 15, 850-859.

(2011): "Robust Performance Hypothesis Testing with the Variance," Wilmott Magazine, forthcoming.

Leland, H. E. (1999): "Optimal Portfolio Management with Transaction Costs and Capital Gains Taxes.," Working Paper.

Maclean, L., Y. Zhao, and W. Ziemba (2006): "Dynamic Portfolio Selection with Process Control," Journal of Banking and Finance, 30 (2), 317-339.

Markowitz, H. (1952): "Portfolio Selection," Journal of Finance, 7, 77-91.

Markowitz, H., And E. L. van DiJK (2003): "Single-Period Mean: Variance Analysis in a Changing World," Financial Analysts Journal, 59, 30-44.

Plaxco, L. M., and R. D. Arnott (2002): "Rebalancing a Global Policy Benchmark," Journal of Portfolio Management, 28, 9-22.

Politis, D. N., And J. P. Romano (1992): "A Circular Block-Resampling Procedure for Stationary Data," in Exploring the Limits of Bootstrap, ed. by R. LePage, and L. Billard, pp. 263-270. John Wiley, New York.

Schreiner, J. (1980): "Portfolio Revision: A Turnover-Constrained Approach," Financial Management, 9, 67-75. 
Sharpe, W. F. (1967): "Portfolio Analysis," Journal of Financial and Quantitative Analysis, 2, 76-84.

Siegel, A. F., And A. Woodgate (2007): "Performance of Portfolios Optimized with Estimation Error," Management Science, 53, 1005-1015.

Tokat, Y., And N. Wicas (2007): "Portfolio Rebalancing in Theory and Practice," Journal of Investing, 16, 52-59.

Tu, J., And G. Zhou (2011): "Markowitz Meets Talmud: A Combination of Sophisticated and Naive Diversification Strategies," Journal of Financial Economics, 99, 204215. 
Table 1: List of considered portfolio strategies

This table lists the minimum-variance portfolio strategies used to evaluate our portfolio revision policy.

Abbreviation

1 Minimum-variance portfolio based on the sample covariance matrix

SAMPLE

2 Minimum-variance portfolio based on the sample covariance matrix with short-sale constraints

Min-C

3 Minimum-variance portfolio based on covariance matrix implied by the single-index model

4 Minimum-variance portfolio as a weighted average of the sample covariance matrix and the covariance matrix implied by the singleindex model

5 Minimum-variance portfolio as a weighted average of the sample covariance matrix and a scalar multiple of the identity matrix

6 Minimum-variance portfolio as a weighted average between the sample covariance matrix and the covariance matrix implied by the constant correlation model 
Table 2: Simulation results for variances

This table reports the variances of the various portfolio strategies revised according to the revision policies derived from the simulation study. The estimation periods comprise $\tau=\{60,120,240,480,960\}$ observations. We compute the $p$-values using Ledoit and Wolf's (2011) studentized circular block bootstrap for the null hypothesis that the variance of our optimal revision policy, $O p t$, is equal to the variance of a particular benchmark revision policy, $n$, i.e., $H_{0}: \hat{\sigma}_{O p t}^{2}-\hat{\sigma}_{n}^{2}=0$. ${ }^{* * *},{ }^{* *}$, and ${ }^{*}$ denote significant differences at the 1,5 , and 10 percent level, respectively.

\begin{tabular}{|c|c|c|c|c|c|c|}
\hline \multirow{2}{*}{$\begin{array}{l}\text { Portfolio } \\
\text { Strategy }\end{array}$} & \multirow{2}{*}{$\begin{array}{c}\text { Revision } \\
\text { Policy }\end{array}$} & \multicolumn{5}{|c|}{ Estimation period length $(\tau)$} \\
\hline & & 60 & 120 & 240 & 480 & 960 \\
\hline \multirow[t]{4}{*}{ SAMPLE } & Opt & 0.00267 & 0.00212 & 0.00194 & 0.00185 & 0.00182 \\
\hline & $1 \mathrm{M}$ & $0.00310^{* * *}$ & $0.00226^{* * *}$ & $0.00200 * * *$ & $0.00189 * * *$ & $0.00183^{* * *}$ \\
\hline & $6 \mathrm{M}$ & $0.00311^{* * *}$ & $0.00226^{* * *}$ & $0.00200^{* * *}$ & $0.00189^{* * *}$ & $0.00183^{* * *}$ \\
\hline & $12 \mathrm{M}$ & $0.00312^{* * *}$ & $0.00226^{* * *}$ & $0.00200 * * *$ & $0.00189 * * *$ & $0.00183^{* * *}$ \\
\hline \multirow[t]{4}{*}{ Min-C } & Opt & 0.00219 & 0.00209 & 0.00202 & 0.00198 & 0.00196 \\
\hline & $1 \mathrm{M}$ & $0.00224^{* * *}$ & $0.00211^{* * *}$ & $0.00203^{* * *}$ & $0.00199 * * *$ & $0.00196^{* *}$ \\
\hline & $6 \mathrm{M}$ & $0.00224^{* * *}$ & $0.00211^{* * *}$ & $0.00203^{* * *}$ & $0.00199 * * *$ & $0.00196^{* *}$ \\
\hline & $12 \mathrm{M}$ & $0.00224^{* * *}$ & $0.00211^{* * *}$ & $0.00203^{* * *}$ & $0.00199 * * *$ & $0.00196^{* *}$ \\
\hline \multirow[t]{4}{*}{$1 \mathrm{~F}$} & Opt & 0.00215 & 0.00209 & 0.00207 & 0.00204 & 0.00207 \\
\hline & $1 \mathrm{M}$ & $0.00223^{* * *}$ & $0.00218^{* * *}$ & 0.00216 *** & $0.00215^{* * *}$ & $0.00213^{* * *}$ \\
\hline & $6 \mathrm{M}$ & $0.00224^{* * *}$ & $0.00218^{* * *}$ & $0.00216^{* * *}$ & $0.00215^{* * *}$ & $0.00213^{* * *}$ \\
\hline & $12 \mathrm{M}$ & $0.00224^{* * *}$ & $0.00218^{* * *}$ & $0.00216^{* * *}$ & $0.00215^{* * *}$ & $0.00213^{* * *}$ \\
\hline \multirow[t]{4}{*}{ LW1F } & Opt & 0.00214 & 0.00201 & 0.00192 & 0.00184 & 0.00182 \\
\hline & $1 \mathrm{M}$ & $0.00220^{* * *}$ & $0.00206^{* * *}$ & $0.00195^{* * *}$ & $0.00188^{* * *}$ & $0.00183^{* * *}$ \\
\hline & $6 \mathrm{M}$ & $0.00221^{* * *}$ & $0.00206^{* * *}$ & $0.00195^{* * *}$ & $0.00188^{* * *}$ & $0.00183^{* * *}$ \\
\hline & $12 \mathrm{M}$ & $0.00221^{* * *}$ & $0.00206^{* * *}$ & $0.00195^{* * *}$ & $0.00188^{* * *}$ & $0.00183^{* * *}$ \\
\hline \multirow[t]{4}{*}{ LWID } & Opt & 0.00227 & 0.00205 & 0.00193 & 0.00185 & 0.00182 \\
\hline & $1 \mathrm{M}$ & $0.00237^{* * *}$ & $0.00213^{* * *}$ & $0.00197^{* * *}$ & $0.00189 * * *$ & $0.00183^{* * *}$ \\
\hline & $6 \mathrm{M}$ & $0.00237^{* * *}$ & $0.00213^{* * *}$ & $0.00197^{* * *}$ & $0.00189 * * *$ & $0.00183^{* * *}$ \\
\hline & $12 \mathrm{M}$ & $0.00238^{* * *}$ & $0.00213^{* * *}$ & $0.00197^{* * *}$ & $0.00189 * * *$ & $0.00183^{* * *}$ \\
\hline \multirow[t]{4}{*}{ LWCC } & Opt & 0.00225 & 0.00205 & 0.00194 & 0.00185 & 0.00182 \\
\hline & $1 \mathrm{M}$ & $0.00232^{* * *}$ & $0.00212^{* * *}$ & $0.00198 * * *$ & $0.00189 * * *$ & $0.00183^{* * *}$ \\
\hline & $6 \mathrm{M}$ & $0.00232^{* * *}$ & $0.00212^{* * *}$ & $0.00197^{* * *}$ & $0.00189^{* * *}$ & $0.00183^{* * *}$ \\
\hline & $12 \mathrm{M}$ & $0.00232^{* * *}$ & $0.00212^{* * *}$ & $0.00197^{* * *}$ & $0.00189 * * *$ & $0.00183^{* * *}$ \\
\hline
\end{tabular}


Table 3: Simulation results for Sharpe ratios

This table reports the Sharpe ratios of the various portfolio strategies revised according to the revision policies derived from the simulation study. The estimation periods comprise $\tau=\{60,120,240,480,960\}$ observations. We compute the $p$-values using Ledoit and Wolf's (2008) studentized circular block bootstrap for the null hypothesis that the Sharpe ratio of our optimal revision policy, $O p t$, is equal to the Sharpe ratio of a particular benchmark revision policy, $n$, i.e., $H_{0}: S R_{O p t}-S R_{n}=0$. ${ }^{* * *}, * *$, and ${ }^{*}$ denote significant differences at the 1,5 , and 10 percent level, respectively.

\begin{tabular}{|c|c|c|c|c|c|c|}
\hline \multirow{2}{*}{$\begin{array}{l}\text { Portfolio } \\
\text { Strategy }\end{array}$} & \multirow{2}{*}{$\begin{array}{c}\text { Revision } \\
\text { Policy }\end{array}$} & \multicolumn{5}{|c|}{ Estimation period length $(\tau)$} \\
\hline & & 60 & 120 & 240 & 480 & 960 \\
\hline \multirow[t]{4}{*}{ SAMPLE } & Opt & 0.0854 & 0.0865 & 0.0951 & 0.0999 & 0.1002 \\
\hline & $1 \mathrm{M}$ & 0.0800 & 0.0869 & 0.0922 & $0.0964^{* *}$ & $0.0984^{*}$ \\
\hline & $6 \mathrm{M}$ & 0.0807 & 0.0867 & 0.0921 & $0.0968 * *$ & $0.0985^{*}$ \\
\hline & $12 \mathrm{M}$ & 0.0791 & 0.0863 & $0.0918^{*}$ & $0.0965^{* *}$ & $0.0985^{*}$ \\
\hline \multirow[t]{4}{*}{ Min-C } & Opt & 0.1190 & 0.1163 & 0.1186 & 0.1219 & 0.1228 \\
\hline & $1 \mathrm{M}$ & 0.1210 & 0.1187 & 0.1197 & 0.1210 & 0.1222 \\
\hline & $6 \mathrm{M}$ & 0.1199 & 0.1183 & 0.1195 & 0.1212 & 0.1222 \\
\hline & $12 \mathrm{M}$ & 0.1182 & 0.1178 & 0.1193 & 0.1209 & 0.1222 \\
\hline \multirow[t]{4}{*}{$1 \mathrm{~F}$} & Opt & 0.1109 & 0.1032 & 0.1066 & 0.1092 & 0.1100 \\
\hline & $1 \mathrm{M}$ & 0.1095 & 0.1038 & $0.1022^{* *}$ & $0.1019 * * *$ & $0.1010^{* * *}$ \\
\hline & $6 \mathrm{M}$ & 0.1090 & 0.1032 & $0.1020^{* *}$ & $0.1021^{* * *}$ & $0.1010^{* * *}$ \\
\hline & $12 \mathrm{M}$ & 0.1087 & 0.1029 & $0.1015^{* * *}$ & $0.1018^{* * *}$ & $0.1010^{* * *}$ \\
\hline \multirow[t]{4}{*}{ LW1F } & Opt & 0.1091 & 0.1015 & 0.1030 & 0.1035 & 0.1024 \\
\hline & $1 \mathrm{M}$ & 0.1097 & 0.1035 & 0.1019 & $0.1012^{*}$ & $0.1008^{*}$ \\
\hline & $6 \mathrm{M}$ & 0.1094 & 0.1030 & 0.1017 & 0.1016 & 0.1009 \\
\hline & $12 \mathrm{M}$ & 0.1086 & 0.1026 & 0.1011 & $0.1013^{*}$ & 0.1009 \\
\hline \multirow[t]{4}{*}{ LWID } & Opt & 0.1057 & 0.0975 & 0.0998 & 0.1020 & 0.1014 \\
\hline & $1 \mathrm{M}$ & 0.1051 & 0.0985 & 0.0981 & $0.0991^{* *}$ & 0.0996* \\
\hline & $6 \mathrm{M}$ & 0.1055 & 0.0983 & 0.0980 & $0.0995^{*}$ & 0.0998* \\
\hline & $12 \mathrm{M}$ & 0.1039 & 0.0979 & 0.0975 & $0.0992^{*}$ & 0.0998 \\
\hline \multirow[t]{4}{*}{ LWCC } & Opt & 0.1181 & 0.1089 & 0.1065 & 0.1056 & 0.1036 \\
\hline & $1 \mathrm{M}$ & 0.1220 & 0.1108 & 0.1061 & 0.1037 & 0.1021 \\
\hline & $6 \mathrm{M}$ & 0.1219 & 0.1102 & 0.1059 & 0.1040 & 0.1022 \\
\hline & $12 \mathrm{M}$ & 0.1204 & 0.1095 & 0.1055 & 0.1037 & 0.1023 \\
\hline
\end{tabular}


Table 4: Simulation results for certainty equivalent returns

This table reports the certainty equivalent returns of the various portfolio strategies revised according to the revision policies derived from the simulation study. The estimation periods comprise $\tau=\{60,120,240,480,960\}$ observations. We compute the $p$-values using the studentized circular block bootstrap described in the appendix (A.1) for the null hypothesis that the certainty equivalent (CEQ) return of our optimal revision policy, $O p t$, is equal to the certainty equivalent of a particular benchmark revision policy, $n$, i.e., $H_{0}: C E Q_{O p t}-C E Q_{n}=0$. ***,**, and $*$ denote significant differences at the 1,5 , and 10 percent level, respectively.

\begin{tabular}{|c|c|c|c|c|c|c|}
\hline \multirow{2}{*}{$\begin{array}{l}\text { Portfolio } \\
\text { Strategy }\end{array}$} & \multirow{2}{*}{$\begin{array}{c}\text { Revision } \\
\text { Policy }\end{array}$} & \multicolumn{5}{|c|}{ Estimation period length $(\tau)$} \\
\hline & & 60 & 120 & 240 & 480 & 960 \\
\hline \multirow[t]{4}{*}{ SAMPLE } & Opt & -0.0022 & -0.0013 & -0.0006 & -0.0003 & -0.0002 \\
\hline & $1 \mathrm{M}$ & $-0.0032^{* * *}$ & -0.0015 & $-0.0008^{* *}$ & $-0.0005^{* * *}$ & $-0.0003^{* *}$ \\
\hline & $6 \mathrm{M}$ & $-0.0032^{* * *}$ & -0.0015 & $-0.0008^{* *}$ & $-0.0005^{* * *}$ & $-0.0003^{* *}$ \\
\hline & $12 \mathrm{M}$ & $-0.0033^{* * *}$ & $-0.0015^{*}$ & $-0.0008^{* * *}$ & $-0.0005^{* * *}$ & $-0.0003^{* *}$ \\
\hline \multirow[t]{4}{*}{ Min-C } & Opt & 0.0001 & 0.0001 & 0.0002 & 0.0004 & 0.0005 \\
\hline & $1 \mathrm{M}$ & 0.0001 & 0.0001 & 0.0003 & 0.0004 & 0.0005 \\
\hline & $6 \mathrm{M}$ & 0.0000 & 0.0001 & 0.0003 & 0.0004 & 0.0005 \\
\hline & $12 \mathrm{M}$ & -0.0001 & 0.0001 & 0.0002 & 0.0004 & 0.0005 \\
\hline \multirow[t]{4}{*}{$1 \mathrm{~F}$} & Opt & -0.0002 & -0.0005 & -0.0003 & -0.0001 & -0.0001 \\
\hline & $1 \mathrm{M}$ & -0.0004 & -0.0006 & $-0.0006^{* * *}$ & $-0.0006^{* * *}$ & $-0.0006^{* * *}$ \\
\hline & $6 \mathrm{M}$ & $-0.0004^{* *}$ & -0.0006 & $-0.0006^{* * *}$ & $-0.0006^{* * *}$ & $-0.0006^{* * *}$ \\
\hline & $12 \mathrm{M}$ & $-0.0004^{* *}$ & -0.0006 & $-0.0006^{* * *}$ & $-0.0006^{* * *}$ & $-0.0006^{* * *}$ \\
\hline \multirow[t]{4}{*}{ LW1F } & Opt & -0.0003 & -0.0004 & -0.0002 & -0.0001 & -0.0001 \\
\hline & $1 \mathrm{M}$ & -0.0003 & -0.0004 & -0.0003 & $-0.0003^{* *}$ & $-0.0002^{* *}$ \\
\hline & $6 \mathrm{M}$ & -0.0003 & -0.0004 & -0.0003 & $-0.0003^{* *}$ & $-0.0002^{* *}$ \\
\hline & $12 \mathrm{M}$ & -0.0004 & -0.0004 & $-0.0004^{*}$ & $-0.0003^{* * *}$ & $-0.0002^{* *}$ \\
\hline \multirow[t]{4}{*}{ LWID } & Opt & -0.0006 & -0.0007 & -0.0004 & -0.0002 & -0.0002 \\
\hline & $1 \mathrm{M}$ & -0.0008 & -0.0007 & $-0.0005^{*}$ & $-0.0004^{* * *}$ & $-0.0003^{* *}$ \\
\hline & $6 \mathrm{M}$ & -0.0007 & -0.0007 & $-0.0005^{*}$ & $-0.0003^{* * *}$ & $-0.0003^{* *}$ \\
\hline & $12 \mathrm{M}$ & $-0.0008^{*}$ & -0.0008 & $-0.0006^{* *}$ & $-0.0004^{* * *}$ & $-0.0003^{* *}$ \\
\hline \multirow[t]{4}{*}{ LWCC } & Opt & 0.0000 & -0.0002 & -0.0001 & -0.0001 & -0.0001 \\
\hline & $1 \mathrm{M}$ & 0.0001 & -0.0002 & -0.0002 & $-0.0002^{* *}$ & $-0.0002^{* *}$ \\
\hline & $6 \mathrm{M}$ & 0.0001 & -0.0002 & -0.0002 & $-0.0002^{* *}$ & $-0.0002^{*}$ \\
\hline & $12 \mathrm{M}$ & -0.0001 & -0.0002 & -0.0002 & $-0.0002^{* *}$ & $-0.0002^{*}$ \\
\hline
\end{tabular}


Table 5: Simulation results for turnovers

This table reports the turnovers of the various portfolio strategies revised according to the revision policies derived from the simulation study. The estimation periods comprise $\tau=\{60,120,240,480,960\}$ observations. We compute the $p$-values using Politis and Romano's (1992) circular block bootstrap for the null hypothesis that the turnover of our optimal revision policy, Opt, is equal to the turnover of a particular benchmark revision policy, $n$, i.e., $H_{0}: t r n_{O p t}-t r n_{n}=0$. ${ }^{* * *}, * *$, and $*$ denote significant differences at the 1,5 , and 10 percent level, respectively.

\begin{tabular}{|c|c|c|c|c|c|c|}
\hline \multirow{2}{*}{$\begin{array}{l}\text { Portfolio } \\
\text { Strategy }\end{array}$} & \multirow{2}{*}{$\begin{array}{c}\text { Revision } \\
\text { Policy }\end{array}$} & \multicolumn{5}{|c|}{ Estimation period length $(\tau)$} \\
\hline & & 60 & 120 & 240 & 480 & 960 \\
\hline \multirow[t]{4}{*}{ SAMPLE } & Opt & 0.2111 & 0.1234 & 0.0984 & 0.0886 & 0.0869 \\
\hline & $1 \mathrm{M}$ & $0.6344^{* * *}$ & $0.2576^{* * *}$ & $0.1446^{* * *}$ & $0.1054^{* * *}$ & $0.0920 * * *$ \\
\hline & $6 \mathrm{M}$ & $0.4153^{* * *}$ & $0.2002^{* * *}$ & $0.1302^{* * *}$ & $0.1028^{* * *}$ & $0.0917^{* * *}$ \\
\hline & $12 \mathrm{M}$ & $0.3478^{* * *}$ & $0.1804^{* * *}$ & $0.1236^{* * *}$ & $0.1009 * * *$ & $0.0913^{* * *}$ \\
\hline \multirow[t]{4}{*}{ Min-C } & Opt & 0.0617 & 0.0473 & 0.0406 & 0.0386 & 0.0381 \\
\hline & $1 \mathrm{M}$ & $0.1504^{* * *}$ & $0.0869 * * *$ & $0.0562^{* * *}$ & $0.0441^{* * *}$ & $0.0399 * * *$ \\
\hline & $6 \mathrm{M}$ & $0.0971^{* * *}$ & $0.0677 * * *$ & $0.0512^{* * *}$ & $0.0433^{* * *}$ & $0.0399 * * *$ \\
\hline & $12 \mathrm{M}$ & $0.0819^{* * *}$ & $0.0606^{* * *}$ & $0.0485^{* * *}$ & $0.0424^{* * *}$ & $0.0397 * * *$ \\
\hline \multirow[t]{4}{*}{$1 \mathrm{~F}$} & Opt & 0.1202 & 0.1059 & 0.1011 & 0.1012 & 0.1052 \\
\hline & $1 \mathrm{M}$ & $0.2306^{* * *}$ & $0.1558 * * *$ & $0.1250^{* * *}$ & $0.1143^{* * *}$ & $0.1110^{* * *}$ \\
\hline & $6 \mathrm{M}$ & $0.1799 * * *$ & $0.1409^{* * *}$ & $0.1216^{* * *}$ & $0.1138 * * *$ & $0.1110^{* * *}$ \\
\hline & $12 \mathrm{M}$ & $0.1615^{* * *}$ & $0.1335^{* * *}$ & $0.1193^{* * *}$ & $0.1132^{* * *}$ & $0.1109^{* * *}$ \\
\hline \multirow[t]{4}{*}{ LW1F } & Opt & 0.1265 & 0.1026 & 0.0915 & 0.0860 & 0.0859 \\
\hline & $1 \mathrm{M}$ & $0.2613^{* * *}$ & $0.1692^{* * *}$ & $0.1222^{* * *}$ & $0.0994^{* * *}$ & $0.0901 * * *$ \\
\hline & $6 \mathrm{M}$ & $0.1917^{* * *}$ & $0.1426^{* * *}$ & $0.1135^{* * *}$ & $0.0975 * * *$ & $0.0899^{* * *}$ \\
\hline & $12 \mathrm{M}$ & $0.1688^{* * *}$ & $0.1320^{* * *}$ & $0.1091^{* * *}$ & $0.0960 * * *$ & $0.0895^{* * *}$ \\
\hline \multirow[t]{4}{*}{ LWID } & Opt & 0.1417 & 0.1077 & 0.0928 & 0.0859 & 0.0856 \\
\hline & $1 \mathrm{M}$ & $0.3293^{* * *}$ & $0.2015^{* * *}$ & $0.1313^{* * *}$ & $0.1013^{* * *}$ & $0.0903^{* * *}$ \\
\hline & $6 \mathrm{M}$ & $0.2267^{* * *}$ & $0.1605^{* * *}$ & $0.1191^{* * *}$ & $0.0989^{* * *}$ & $0.0901 * * *$ \\
\hline & $12 \mathrm{M}$ & $0.1949 * * *$ & $0.1457 * * *$ & $0.1133^{* * *}$ & $0.0971^{* * *}$ & $0.0897 * * *$ \\
\hline \multirow[t]{4}{*}{ LWCC } & Opt & 0.1160 & 0.0929 & 0.0851 & 0.0819 & 0.0833 \\
\hline & $1 \mathrm{M}$ & $0.2446^{* * *}$ & $0.1669 * * *$ & $0.1186^{* * *}$ & $0.0956^{* * *}$ & $0.0875^{* * *}$ \\
\hline & $6 \mathrm{M}$ & $0.1755^{* * *}$ & $0.1355^{* * *}$ & $0.1082^{* * *}$ & $0.0935^{* * *}$ & $0.0873^{* * *}$ \\
\hline & $12 \mathrm{M}$ & $0.1537^{* * *}$ & $0.1238^{* * *}$ & $0.1031^{* * *}$ & $0.0919 * * *$ & $0.0869^{* * *}$ \\
\hline
\end{tabular}


Table 6: List of data sets

This table lists the various datasets used for evaluating portfolio performance, as well as their abbreviations, the number of assets in each dataset, the time period over which we used data from each set, and the data sources. All datasets are comprised of monthly data, and, for portfolio data, they apply the value-weighting scheme to the respective constituents. Datasets from Kenneth French are taken from his website: http://mba. tuck.dartmouth.edu/pages/faculty/ken.french/data_library.html, and represent different breakdowns of U.S. stock market data. The STOCK dataset comprises single stocks from the Center for Research in Security Prices (CRSP).

\begin{tabular}{llcccc}
\hline$\#$ & Dataset & Abbreviation & N & Time period & Source \\
\hline 1 & $\begin{array}{l}6 \text { Fama and French portfolios } \\
\text { of firms sorted by size and } \\
\text { book-to-market ratio }\end{array}$ & $6 \mathrm{FF}$ & 6 & $07 / 1963-12 / 2008$ & K. French \\
2 & & & & \\
$\quad \begin{array}{l}25 \text { Fama and French portfo- } \\
\text { lios of firms sorted by size and } \\
\text { book-to-market ratio }\end{array}$ & $25 \mathrm{FF}$ & 25 & $07 / 1963-12 / 2008$ & K. French \\
3 & $\begin{array}{l}10 \text { industry portfolios repre- } \\
\text { senting the U.S. stock market }\end{array}$ & 10IND & 10 & $07 / 1963-12 / 2008$ & K. French \\
4 & $\begin{array}{l}48 \text { industry portfolios repre- } \\
\text { senting the U.S. stock market }\end{array}$ & 48IND & 48 & $07 / 1963-12 / 2008$ & K. French \\
$\begin{array}{l}100 \text { Stocks with the largest } \\
\text { average market capitalization }\end{array}$ & STOCK & 100 & $07 / 1963-12 / 2008$ & CRSP \\
\hline
\end{tabular}


Table 7: Empirical results for variances

This table reports the variances of the various portfolio strategies revised according to the revision policies derived from the empirical study. We compute the $p$-values using Ledoit and Wolf's (2011) studentized circular block bootstrap for the null hypothesis that the variance of our optimal revision policy, $O p t$, is equal to the variance of a particular benchmark revision policy, $n$, i.e., $H_{0}: \hat{\sigma}_{O p t}^{2}-\hat{\sigma}_{n}^{2}=0$. ${ }^{* * *}, * *$ and ${ }^{*}$ denote statistical significance at the 1,5 , and 10 percent level, respectively.

\begin{tabular}{|c|c|c|c|c|c|c|}
\hline \multirow{2}{*}{$\begin{array}{l}\text { Portfolio } \\
\text { Strategy }\end{array}$} & \multirow{2}{*}{$\begin{array}{c}\text { Revision } \\
\text { Policy }\end{array}$} & \multicolumn{5}{|c|}{ Dataset } \\
\hline & & $6 \mathrm{FF}$ & $25 \mathrm{FF}$ & 10IND & $48 \mathrm{IND}$ & STOCK \\
\hline \multirow[t]{4}{*}{ SAMPLE } & Opt & 0.00167 & 0.00152 & 0.00138 & 0.00194 & 0.00503 \\
\hline & $1 \mathrm{M}$ & $0.00162^{* *}$ & 0.00149 & 0.00136 & 0.00189 & $0.00679^{* * *}$ \\
\hline & $6 \mathrm{M}$ & 0.00170 & 0.00156 & 0.00138 & 0.00193 & $0.00632^{* * *}$ \\
\hline & $12 \mathrm{M}$ & $0.00174^{*}$ & 0.00157 & 0.00135 & 0.00203 & $0.00675^{* * *}$ \\
\hline \multirow[t]{4}{*}{ Min-C } & Opt & 0.00198 & 0.00186 & 0.00138 & 0.00144 & 0.00153 \\
\hline & $1 \mathrm{M}$ & 0.00201 & 0.00185 & 0.00138 & $0.00140 * *$ & 0.00150 \\
\hline & $6 \mathrm{M}$ & 0.00200 & 0.00187 & 0.00138 & 0.00143 & 0.00152 \\
\hline & $12 \mathrm{M}$ & 0.00201 & 0.00188 & 0.00136 & 0.00143 & 0.00156 \\
\hline \multirow[t]{4}{*}{$1 \mathrm{~F}$} & Opt & 0.00214 & 0.00299 & 0.00144 & 0.00154 & 0.00177 \\
\hline & $1 \mathrm{M}$ & 0.00222 & $0.00315^{* *}$ & 0.00142 & 0.00158 & $0.00245^{* * *}$ \\
\hline & $6 \mathrm{M}$ & $0.00233^{* *}$ & $0.00336^{* *}$ & 0.00144 & 0.00159 & $0.00253^{* * *}$ \\
\hline & $12 \mathrm{M}$ & $0.00243^{*}$ & $0.00351^{* *}$ & 0.00142 & 0.00161 & $0.00247^{* * *}$ \\
\hline \multirow[t]{4}{*}{ LW1F } & Opt & 0.00169 & 0.00143 & 0.00136 & 0.00145 & 0.00157 \\
\hline & $1 \mathrm{M}$ & $0.00164^{* *}$ & 0.00139 & 0.00134 & $0.00138^{* *}$ & $0.00149 *$ \\
\hline & $6 \mathrm{M}$ & 0.00172 & 0.00145 & 0.00135 & 0.00141 & 0.00155 \\
\hline & $12 \mathrm{M}$ & $0.00176^{*}$ & $0.00146^{*}$ & $0.00133^{* *}$ & 0.00145 & 0.00158 \\
\hline \multirow[t]{4}{*}{ LWID } & Opt & 0.00167 & 0.00136 & 0.00133 & 0.00154 & 0.00166 \\
\hline & $1 \mathrm{M}$ & 0.00163 & 0.00133 & $0.00131^{*}$ & $0.00145^{* * *}$ & $0.00158^{*}$ \\
\hline & $6 \mathrm{M}$ & 0.00168 & 0.00139 & 0.00132 & $0.00148^{* *}$ & 0.00165 \\
\hline & $12 \mathrm{M}$ & 0.00171 & $0.00141^{* *}$ & $0.00130 * * *$ & 0.00153 & 0.00172 \\
\hline \multirow[t]{4}{*}{ LWCC } & Opt & 0.00179 & 0.00153 & 0.00136 & 0.00148 & 0.00158 \\
\hline & $1 \mathrm{M}$ & $0.00193^{* * *}$ & $0.00200^{* * *}$ & 0.00133 & $0.00141^{* *}$ & $0.00152^{*}$ \\
\hline & $6 \mathrm{M}$ & $0.00205^{* * *}$ & $0.00215^{* * *}$ & 0.00136 & 0.00146 & 0.00158 \\
\hline & $12 \mathrm{M}$ & $0.00214^{* *}$ & $0.00224^{* * *}$ & 0.00135 & 0.00148 & 0.00159 \\
\hline
\end{tabular}


Table 8: Empirical results for Sharpe ratios

This table reports the Sharpe ratios of the various portfolio strategies revised according to the revision policies derived from the empirical study. We compute the $p$-values using Ledoit and Wolf's (2008) studentized circular block bootstrap for the null hypothesis that the Sharpe ratio of our optimal revision policy, $O p t$, is equal to the Sharpe ratio of a particular benchmark revision policy, $n$, i.e., $H_{0}: S R_{O p t}-S R_{n}=0$. ****** and ${ }^{*}$ denote statistical significance at the 1,5 , and 10 percent level, respectively.

\begin{tabular}{|c|c|c|c|c|c|c|}
\hline \multirow{2}{*}{$\begin{array}{l}\text { Portfolio } \\
\text { Strategy }\end{array}$} & \multirow{2}{*}{$\begin{array}{c}\text { Revision } \\
\text { Policy }\end{array}$} & \multicolumn{5}{|c|}{ Dataset } \\
\hline & & $6 \mathrm{FF}$ & $25 \mathrm{FF}$ & 10IND & $48 \mathrm{IND}$ & STOCK \\
\hline \multirow[t]{4}{*}{ SAMPLE } & Opt & 0.2070 & 0.2345 & 0.1393 & 0.0814 & 0.0162 \\
\hline & $1 \mathrm{M}$ & 0.2084 & 0.2438 & 0.1451 & $0.0568^{*}$ & -0.010 \\
\hline & $6 \mathrm{M}$ & 0.2064 & 0.2378 & 0.1434 & $0.0485^{* *}$ & -0.017 \\
\hline & $12 \mathrm{M}$ & 0.2069 & 0.2496 & 0.1378 & $0.0513^{* *}$ & $-0.032^{*}$ \\
\hline \multirow[t]{4}{*}{ Min-C } & Opt & 0.1228 & 0.1214 & 0.1377 & 0.1259 & 0.1043 \\
\hline & $1 \mathrm{M}$ & 0.1202 & 0.1230 & 0.1415 & 0.1347 & 0.1018 \\
\hline & $6 \mathrm{M}$ & 0.1204 & 0.1233 & 0.1412 & 0.1306 & 0.0982 \\
\hline & $12 \mathrm{M}$ & 0.1202 & 0.1275 & 0.1404 & 0.1333 & 0.0991 \\
\hline \multirow[t]{4}{*}{$1 \mathrm{~F}$} & Opt & 0.1080 & 0.1184 & 0.1524 & 0.1179 & 0.0943 \\
\hline & $1 \mathrm{M}$ & 0.0987 & 0.1216 & 0.1651 & 0.1015 & 0.0614 \\
\hline & $6 \mathrm{M}$ & 0.0985 & 0.1216 & 0.1631 & 0.1026 & $0.0608^{*}$ \\
\hline & $12 \mathrm{M}$ & 0.1008 & 0.1198 & 0.1586 & 0.1057 & 0.0623 \\
\hline \multirow[t]{4}{*}{ LW1F } & Opt & 0.1947 & 0.2201 & 0.1422 & 0.0932 & 0.0673 \\
\hline & $1 \mathrm{M}$ & 0.1955 & 0.2300 & 0.1473 & 0.0798 & 0.0629 \\
\hline & $6 \mathrm{M}$ & 0.1931 & 0.2250 & 0.1458 & 0.0809 & 0.0619 \\
\hline & $12 \mathrm{M}$ & 0.1936 & 0.2316 & 0.1398 & 0.0836 & 0.0677 \\
\hline \multirow[t]{4}{*}{ LWID } & Opt & 0.1669 & 0.2116 & 0.1388 & 0.1051 & 0.0524 \\
\hline & $1 \mathrm{M}$ & 0.1617 & 0.2107 & 0.1464 & 0.0938 & 0.0507 \\
\hline & $6 \mathrm{M}$ & 0.1587 & 0.2066 & 0.1458 & 0.0945 & 0.0553 \\
\hline & $12 \mathrm{M}$ & 0.1603 & 0.2095 & 0.1410 & 0.0961 & 0.0633 \\
\hline \multirow[t]{4}{*}{ LWCC } & Opt & 0.1413 & 0.1926 & 0.1362 & 0.0984 & 0.0519 \\
\hline & $1 \mathrm{M}$ & $0.1200^{* *}$ & 0.1672 & 0.1400 & 0.0895 & 0.0458 \\
\hline & $6 \mathrm{M}$ & $0.1208^{*}$ & 0.1716 & 0.1423 & 0.0900 & 0.0455 \\
\hline & $12 \mathrm{M}$ & 0.1235 & 0.1752 & 0.1356 & 0.0940 & 0.0545 \\
\hline
\end{tabular}


Table 9: Empirical results for certainty equivalent returns

This table reports the certainty equivalent returns of the various portfolio strategies revised according to the revision policies derived from the empirical study. We compute the $p$-values using the studentized circular block bootstrap described in the appendix A.1 for the null hypothesis that the certainty equivalent (CEQ) return of our optimal revision policy, $O p t$, is equal to the certainty equivalent of a particular benchmark revision policy, $n$, i.e., $H_{0}: C E Q_{O p t}-C E Q_{n}=0 .{ }^{* * *}{ }^{* *}$, and ${ }^{*}$ denote significant differences at the 1 , 5 , and 10 percent level, respectively.

\begin{tabular}{|c|c|c|c|c|c|c|}
\hline \multirow{2}{*}{$\begin{array}{l}\text { Portfolio } \\
\text { Strategy }\end{array}$} & \multirow{2}{*}{$\begin{array}{c}\text { Revision } \\
\text { Policy }\end{array}$} & \multicolumn{5}{|c|}{ Dataset } \\
\hline & & $6 \mathrm{FF}$ & $25 \mathrm{FF}$ & 10IND & 48IND & STOCK \\
\hline \multirow[t]{4}{*}{ SAMPLE } & Opt & 0.0042 & 0.0053 & 0.0017 & -0.0012 & -0.0114 \\
\hline & $1 \mathrm{M}$ & 0.0043 & 0.0056 & 0.0019 & -0.0022 & $-0.0178^{* *}$ \\
\hline & $6 \mathrm{M}$ & 0.0042 & 0.0054 & 0.0018 & $-0.0027^{* *}$ & $-0.0172^{* *}$ \\
\hline & $12 \mathrm{M}$ & 0.0042 & 0.0059 & 0.0016 & $-0.0027^{* *}$ & $-0.0196^{* * *}$ \\
\hline \multirow[t]{4}{*}{ Min-C } & Opt & 0.0005 & 0.0005 & 0.0016 & 0.0011 & 0.0002 \\
\hline & $1 \mathrm{M}$ & 0.0003 & 0.0006 & 0.0018 & 0.0015 & 0.0001 \\
\hline & $6 \mathrm{M}$ & 0.0003 & 0.0006 & 0.0017 & 0.0013 & 0.0000 \\
\hline & $12 \mathrm{M}$ & 0.0003 & 0.0008 & 0.0017 & 0.0014 & 0.0000 \\
\hline \multirow[t]{4}{*}{$1 \mathrm{~F}$} & Opt & -0.0003 & -0.0010 & 0.0021 & 0.0007 & -0.0004 \\
\hline & $1 \mathrm{M}$ & -0.0009 & -0.0010 & 0.0026 & 0.0001 & $-0.0030 * *$ \\
\hline & $6 \mathrm{M}$ & -0.0010 & -0.0013 & 0.0025 & 0.0001 & $-0.0032^{* *}$ \\
\hline & $12 \mathrm{M}$ & -0.0011 & -0.0016 & 0.0024 & 0.0002 & $-0.0030^{* *}$ \\
\hline \multirow[t]{4}{*}{ LW1F } & Opt & 0.0037 & 0.0047 & 0.0018 & 0.0000 & -0.0012 \\
\hline & $1 \mathrm{M}$ & 0.0038 & 0.0051 & 0.0020 & -0.0004 & -0.0012 \\
\hline & $6 \mathrm{M}$ & 0.0037 & 0.0049 & 0.0019 & -0.0004 & -0.0014 \\
\hline & $12 \mathrm{M}$ & 0.0037 & 0.0052 & 0.0017 & -0.0004 & -0.0012 \\
\hline \multirow[t]{4}{*}{ LWID } & Opt & 0.0026 & 0.0044 & 0.0017 & 0.0002 & -0.0020 \\
\hline & $1 \mathrm{M}$ & 0.0024 & 0.0043 & 0.0020 & -0.0001 & -0.0019 \\
\hline & $6 \mathrm{M}$ & 0.0023 & 0.0042 & 0.0019 & -0.0001 & -0.0018 \\
\hline & $12 \mathrm{M}$ & 0.0023 & 0.0043 & 0.0018 & -0.0001 & -0.0016 \\
\hline \multirow[t]{4}{*}{ LWCC } & Opt & 0.0015 & 0.0037 & 0.0016 & -0.0000 & -0.0018 \\
\hline & $1 \mathrm{M}$ & $0.0004^{* *}$ & 0.0024 & 0.0017 & -0.0001 & -0.0020 \\
\hline & $6 \mathrm{M}$ & $0.0003^{* *}$ & 0.0025 & 0.0018 & -0.0002 & -0.0021 \\
\hline & $12 \mathrm{M}$ & 0.0003 & 0.0026 & 0.0016 & -0.0001 & -0.0018 \\
\hline
\end{tabular}


Table 10: Empirical results for turnovers

This table reports the turnovers of the various portfolio strategies revised according to the revision policies derived from the empirical study. We compute the $p$-values using Politis and Romano's (1992) circular block bootstrap for the null hypothesis that the turnover of our optimal revision policy, $O p t$, is equal to the turnover of a particular benchmark revision policy, $n$, i.e., $H_{0}: t r n_{O p t}-t r n_{n}=0 . * * * * *$, and ${ }^{*}$ denote significant differences at the 1,5 , and 10 percent level, respectively.

\begin{tabular}{|c|c|c|c|c|c|c|}
\hline \multirow{2}{*}{$\begin{array}{l}\text { Portfolio } \\
\text { Strategy }\end{array}$} & \multirow{2}{*}{$\begin{array}{c}\text { Revision } \\
\text { Policy }\end{array}$} & \multicolumn{5}{|c|}{ Dataset } \\
\hline & & $6 \mathrm{FF}$ & $25 \mathrm{FF}$ & 10IND & $48 I N D$ & STOCK \\
\hline \multirow[t]{4}{*}{ SAMPLE } & Opt & 0.1408 & 0.3539 & 0.0881 & 0.3359 & 0.9925 \\
\hline & $1 \mathrm{M}$ & $0.2215^{* * *}$ & $0.7868^{* * *}$ & $0.1604^{* * *}$ & $0.8066^{* * *}$ & $3.4585^{* * *}$ \\
\hline & $6 \mathrm{M}$ & $0.1828 * * *$ & $0.5518^{* * *}$ & $0.1207^{* * *}$ & $0.5389 * * *$ & $2.0778^{* * *}$ \\
\hline & $12 \mathrm{M}$ & $0.1715^{* * *}$ & $0.4764^{* * *}$ & $0.1023^{* *}$ & $0.4624^{* * *}$ & $1.7010^{* * *}$ \\
\hline \multirow[t]{4}{*}{ Min-C } & Opt & 0.0143 & 0.0226 & 0.0283 & 0.0434 & 0.0641 \\
\hline & $1 \mathrm{M}$ & $0.0290 * *$ & $0.1007^{* * *}$ & $0.0773^{* * *}$ & $0.1172^{* * *}$ & $0.1598^{* * *}$ \\
\hline & $6 \mathrm{M}$ & $0.0206^{* * *}$ & $0.0416^{* * *}$ & $0.0397^{* * *}$ & $0.0558 * * *$ & $0.0892^{* * *}$ \\
\hline & $12 \mathrm{M}$ & $0.0201^{* *}$ & $0.0334^{* * *}$ & $0.0365^{* * *}$ & 0.0472 & $0.0755^{*}$ \\
\hline \multirow[t]{4}{*}{$1 \mathrm{~F}$} & Opt & 0.0844 & 0.1470 & 0.0680 & 0.1352 & 0.1158 \\
\hline & $1 \mathrm{M}$ & $0.1218^{* * *}$ & $0.2379 * * *$ & $0.0997^{* * *}$ & $0.1874 * * *$ & $0.1730^{* * *}$ \\
\hline & $6 \mathrm{M}$ & $0.1168 * * *$ & $0.2328 * * *$ & $0.0885^{* * *}$ & $0.1733^{* * *}$ & $0.1624^{* * *}$ \\
\hline & $12 \mathrm{M}$ & $0.1155^{* * *}$ & $0.2309 * * *$ & $0.0821^{* * *}$ & $0.1655^{* * *}$ & $0.1539^{* * *}$ \\
\hline \multirow[t]{4}{*}{ LW1F } & Opt & 0.1262 & 0.2850 & 0.0834 & 0.1968 & 0.2174 \\
\hline & $1 \mathrm{M}$ & $0.1990^{* * *}$ & $0.5600^{* * *}$ & $0.1416^{* * *}$ & $0.3643^{* * *}$ & $0.3615^{* * *}$ \\
\hline & $6 \mathrm{M}$ & $0.1638^{* * *}$ & $0.4115^{* * *}$ & $0.1096^{* * *}$ & $0.2694^{* * *}$ & $0.2781^{* * *}$ \\
\hline & $12 \mathrm{M}$ & $0.1535^{* * *}$ & $0.3613^{* * *}$ & $0.0934^{*}$ & $0.2391^{* * *}$ & $0.2504^{* *}$ \\
\hline \multirow[t]{4}{*}{ LWID } & Opt & 0.0689 & 0.1985 & 0.0670 & 0.2133 & 0.2640 \\
\hline & $1 \mathrm{M}$ & $0.0899 * * *$ & $0.3138^{* * *}$ & $0.1097^{* * *}$ & $0.3957^{* * *}$ & $0.4857^{* * *}$ \\
\hline & $6 \mathrm{M}$ & $0.0804^{* *}$ & $0.2430 * * *$ & $0.0869^{* * *}$ & $0.2848 * * *$ & $0.3586^{* * *}$ \\
\hline & $12 \mathrm{M}$ & 0.0757 & 0.2178 & 0.0744 & $0.2517^{* *}$ & $0.3203^{* * *}$ \\
\hline \multirow[t]{4}{*}{ LWCC } & Opt & 0.0635 & 0.1618 & 0.0627 & 0.1851 & 0.1885 \\
\hline & $1 \mathrm{M}$ & $0.1067^{* * *}$ & $0.2895^{* * *}$ & $0.1005^{* * *}$ & $0.3292^{* * *}$ & $0.2872^{* * *}$ \\
\hline & $6 \mathrm{M}$ & $0.0969 * * *$ & $0.2483^{* * *}$ & $0.0815^{* * *}$ & $0.2461^{* * *}$ & $0.2313^{* * *}$ \\
\hline & $12 \mathrm{M}$ & $0.0925^{* * *}$ & $0.2313^{* * *}$ & 0.0701 & $0.2184^{* *}$ & $0.2105^{*}$ \\
\hline
\end{tabular}


Figure 1: Revision intensities for the STOCK dataset

The six panels in this figure show the fraction of wealth, $\alpha$, that is retained in the old portfolio weights, $w_{t-1}^{R e v}$, over the sample period from 08/1973 - 12/2008 for the six considered minimum-variance portfolio strategies.
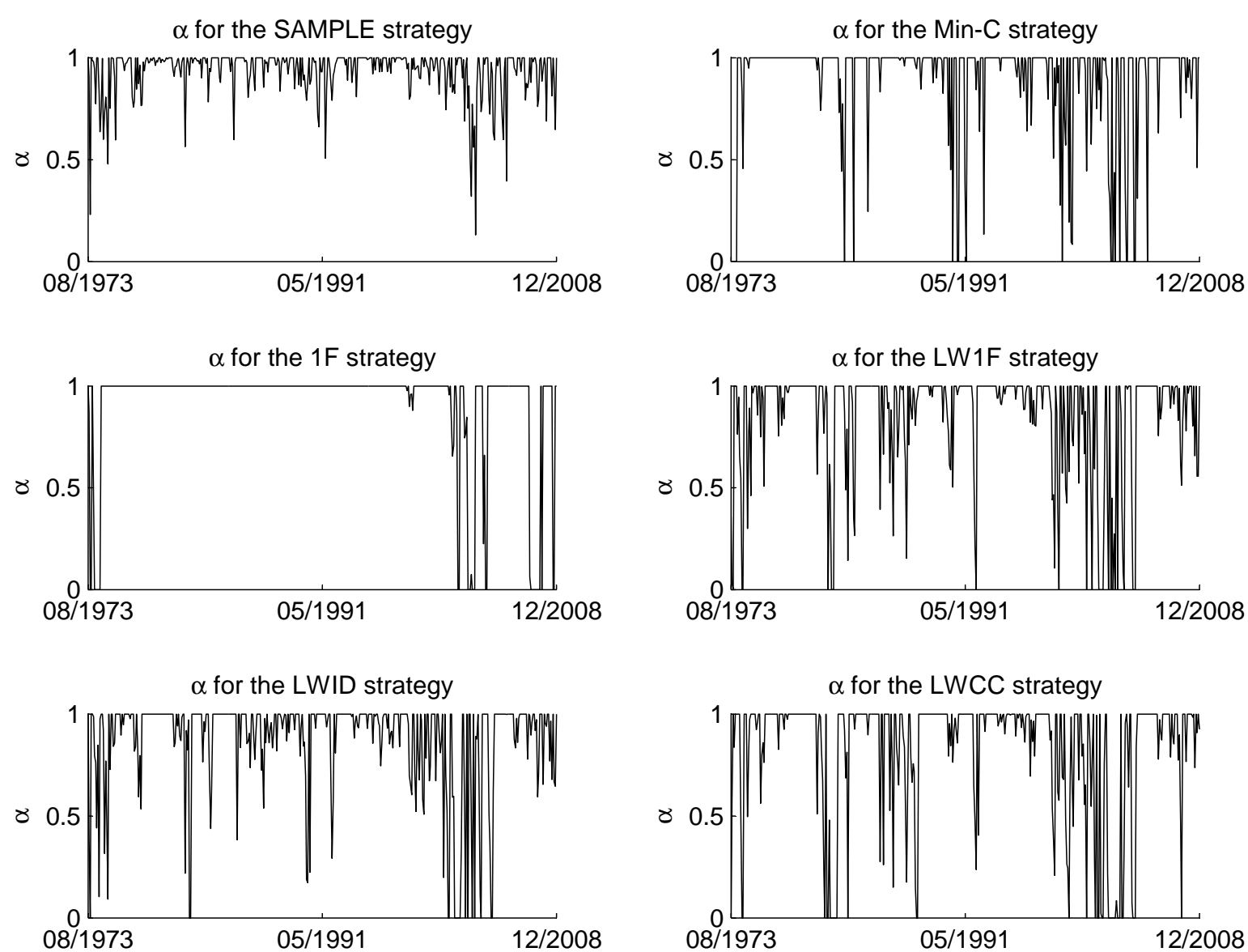
Figure 2: Sharpe ratio differences for the STOCK dataset

The eighteen panels in this figure show the Sharpe ratio differences ( $\Delta \mathrm{SR})$ between our proposed revision policy and the fixed revision frequencies of one, six, and twelve months as a function of proportional oneway transaction costs in basis points (bps). The Sharpe ratio differences are shown by dashed lines, while the dotted lines represent the $90 \%$ confidence interval. For this interval, we use bootstrapped standard errors obtained from Ledoit and Wolf's (2008) studentized circular block bootstrap.
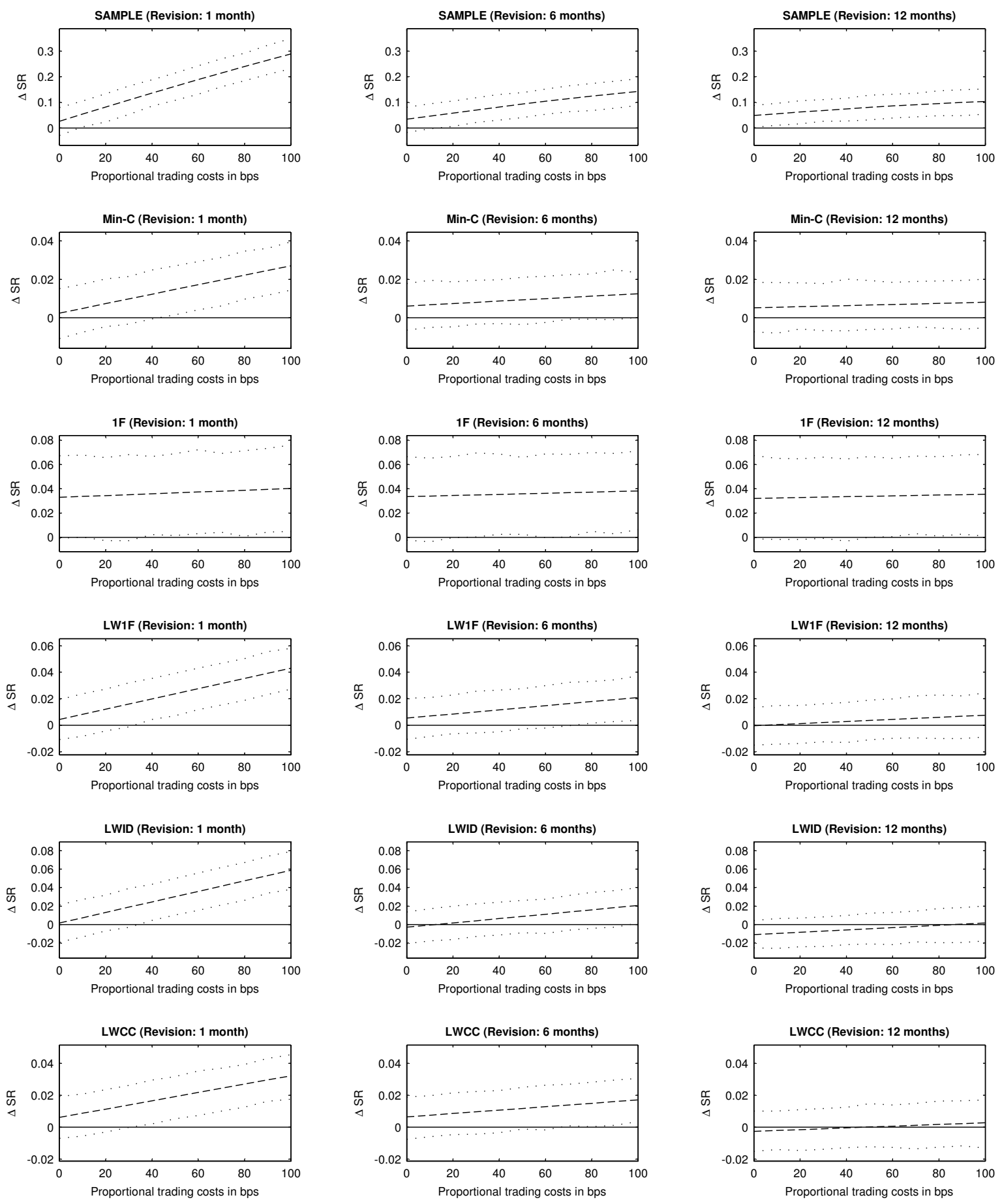


\section{Figure 3: Certainty equivalent return differences for the STOCK dataset}

The eighteen panels in this figure show the certainty equivalent return differences ( $\Delta$ CEQ) between our proposed revision policy and the fixed revision frequencies of one, six, and twelve months as a function of proportional one-way transaction costs in basis points (bps). The certainty equivalent return differences are shown by dashed lines, while the dotted lines represent the $90 \%$ confidence interval. For this interval, we use bootstrapped standard errors obtained from the studentized circular block bootstrap described in appendix A.1.
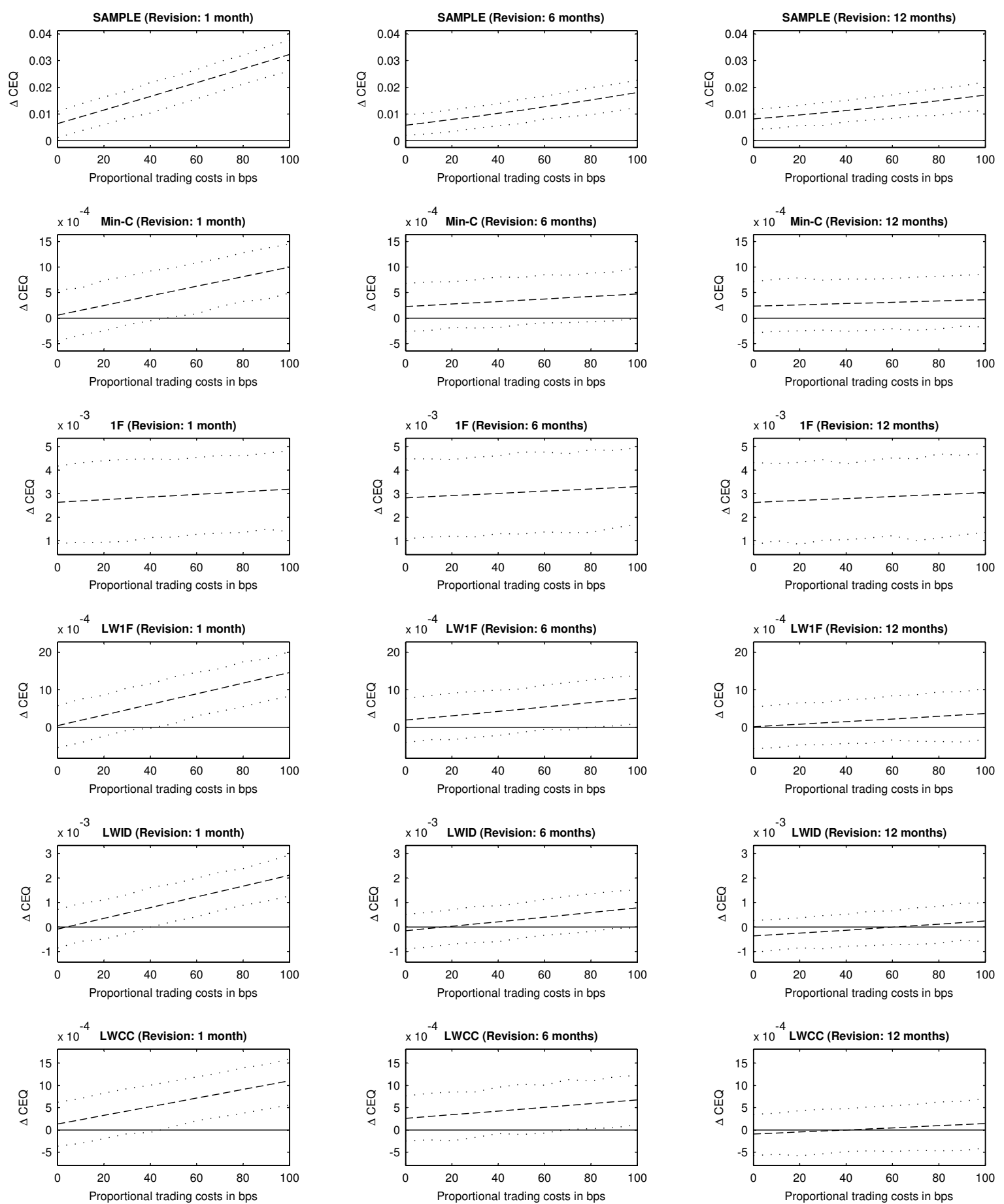